\title{
Quantizations on Nilpotent Lie Groups and Algebras Having Flat Coadjoint Orbits
}

\author{
M. Măntoiu and M. Ruzhansky *
}

\begin{abstract}
For a connected simply connected nilpotent Lie group $\mathrm{G}$ with Lie algebra $\mathfrak{g}$ and unitary dual $\widehat{\mathrm{G}}$ one has (a) a global quantization of operator-valued symbols defined on $\mathrm{G} \times \widehat{\mathrm{G}}$, involving the representation theory of the group, (b) a quantization of scalar-valued symbols defined on $G \times \mathfrak{g}^{*}$, taking the group structure into account and (c) Weyl-type quantizations of all the coadjoint orbits $\left\{\Omega_{\xi} \mid \xi \in \widehat{G}\right\}$. We show how these quantizations are connected, in the case when flat coadjoint orbits exist. This is done by a careful analysis of the composition of two different types of Fourier transformations. We also describe the concrete form of the operator-valued symbol quantization, by using Kirillov theory and the Euclidean version of the unitary dual and Plancherel measure. In the case of the Heisenberg group this corresponds to the known picture, presenting the representation theoretical pseudo-differential operators in terms of families of Weyl operators depending on a parameter. For illustration, we work out a couple of examples and put into evidence some specific features of the case of Lie algebras with one-dimensional center. When $\mathrm{G}$ is also graded, we make a short presentation of the symbol classes $S_{\rho, \delta}^{m}$, transferred from $\mathrm{G} \times \widehat{\mathrm{G}}$ to $G \times \mathfrak{g}^{*}$ by means of the connection mentioned above.
\end{abstract}

\section{Introduction}

The article treats pseudo-differential operators associated to a connected simply connected nilpotent Lie group $\mathrm{G}$ with Haar measure $d \mathrm{~m}(x)$ and Lie algebra $\mathfrak{g}$. Denoting by $\mathfrak{g}^{*}$ the dual of $\mathfrak{g}$ and by $\widehat{\mathrm{G}}$ the unitary dual of $\mathrm{G}$, composed of unitary equivalence classses of irreducible representations, one has various pseudo-differential calculi:

1. A global quantization $O \mathrm{p}_{\mathrm{G} \times \widehat{\mathrm{G}}}$ of operator-valued symbols $[10,11,12,26]$ defined on $\mathrm{G} \times \widehat{\mathrm{G}}$, making strong use of the representation theory of the group (see [34,35] for the compact Lie group case). As an outcome, we get operators acting in function spaces defined on $\mathrm{G}$, as $L^{2}(\mathrm{G})$ for instance.

2. A quantization $\mathrm{Op}_{\mathrm{G} \times \mathfrak{g}^{*}}$ of scalar-valued symbols defined on $\mathrm{G} \times \mathfrak{g}^{*}$, taking the group law into account and different from the usual cotangent bundle quantization. Once again one gets operators acting in function spaces defined on $G$.

3. Quantizations $\operatorname{Ped}_{\xi}$ [32] of all the coadjoint orbits $\left\{\Omega_{\xi} \subset \mathfrak{g}^{*} \mid \xi \in \widehat{\mathrm{G}}\right\}$. This generalizes the Weyl calculus, seen as a pseudo-differential theory on the coadjoint orbits of the Heisenberg group. The emerging operators act on the Hilbert space $\mathcal{H}_{\xi}$ of the irreducible representation $\xi$ or on the corresponding space $\mathcal{H}_{\xi}^{\infty}$ of smooth vectors.

*2010 Mathematics Subject Classification: Primary 22E25, 47G30, Secundary 22E30.

Key Words: nilpotent group, Lie algebra, coadjoint orbit, pseudo-differential operator, symbol, Weyl calculus M. M. has been supported by Núcleo Milenio de Física Matemática RC120002 and the Fondecyt Project 1160359. M. R. was supported in parts by the EPSRC grant EP/K039407/1 and by the Leverhulme Grant RPG-2014-02. 
To make things clear, let us indicate the basic formulae for $\mathrm{Op}_{\mathrm{G} \times \mathfrak{g}^{*}}$ and $O \mathrm{p}_{\mathrm{G} \times \widehat{G}}$.

Recall first that for connected simply connected nilpotent Lie groups the exponential map exp $: \mathfrak{g} \rightarrow \mathrm{G}$ is a global diffeomorphism; its inverse will be denoted by $\log$. Since $\mathfrak{g}$ and $\mathfrak{g}^{*}$ are dual finite-dimensional vector spaces, one can start with the familiar Kohn-Nirenberg formula

$$
\left[\mathfrak{o} \mathfrak{p}_{\mathfrak{g} \times \mathfrak{g}^{*}}(\mathfrak{f}) \nu\right](X)=\int_{\mathfrak{g}} \int_{\mathfrak{g}^{*}} e^{i\langle X-Y \mid \mathcal{X}\rangle} \mathfrak{f}(X, \mathcal{X}) \nu(Y) d Y d \mathcal{X}
$$

associating to certain functions $\mathfrak{f}: \mathfrak{g} \times \mathfrak{g}^{*} \rightarrow \mathbb{C}$ operators acting on $\nu \in L^{2}(\mathfrak{g})$. Then, suitably composing with the functions exp and $\log$ at the level of the vectors or of the symbols, one easily arrives at the formula

$$
\left[\operatorname{op}_{\mathrm{G} \times \mathfrak{g}^{*}}(f) u\right](x)=\int_{\mathrm{G}} \int_{\mathfrak{g}^{*}} e^{i\langle\log x-\log y \mid \mathcal{X}\rangle} f(x, \mathcal{X}) u(y) d \mathrm{~m}(y) d \mathcal{X}
$$

in which $f: G \times \mathfrak{g}^{*} \rightarrow \mathbb{C}$ and $u: G \rightarrow \mathbb{C}$ are suitable functions. Although $G \times \mathfrak{g}^{*}$ can be identified with the cotangent bundle of the manifold $G$, it is clear that the group structure of $G$ (or the Lie algebra structure of $\mathfrak{g}$ ) plays no role and this makes (1.2) unsuitable for our purposes. In (1.1), for instance, if $f$ only depends on $\mathcal{X} \in \mathfrak{g}^{*}$, the operator $\mathfrak{o p}_{\mathfrak{g} \times \mathfrak{g}^{*}}(\mathfrak{f})$ is just a convolution by the Euclidean Fourier transform of $\mathrm{f}$. The group involved in this convolution is just $(\mathfrak{g},+)$, leading to a commutative convolution calculus, and this is not what we want.

A better situation occurs if on $\mathfrak{g}$, instead of the vector sum, one considers the group operation $(X, Y) \mapsto$ $X \bullet Y:=\log [\exp (X) \exp (Y)]$ obtained from $\mathrm{G}$ by transport of structure; it is given by the Baker-CampbellHausdorff formula, consisting in our nilpotent case of a finite combination of successive commutators of $X$ and $Y$. We are lead to replace (1.1) by

$$
\left[\mathfrak{O p}_{\mathfrak{g} \times \mathfrak{g}^{*}}(\mathfrak{f}) \nu\right](X)=\int_{\mathfrak{g}} \int_{\mathfrak{g}^{*}} e^{i\langle(-Y) \bullet X \mid \mathcal{X}\rangle} \mathrm{f}(X, \mathcal{X}) \nu(Y) d Y d \mathcal{X}
$$

Performing the same compositions with exp and log, one finally gets instead of (1.2) the quantization formula

$$
\left[\mathrm{Op}_{\mathrm{G} \times \mathfrak{g}^{*}}(f) u\right](x)=\int_{\mathrm{G}} \int_{\mathfrak{g}^{*}} e^{i\left\langle\log \left(y^{-1} x\right) \mid \mathcal{X}\right\rangle} f(x, \mathcal{X}) u(y) d \mathrm{~m}(y) d \mathcal{X} .
$$

To be precise, the needed transformations are $\nu \mapsto u:=\nu \circ \log$ and $\mathrm{f} \mapsto f:=\mathrm{f} \circ\left(\log \otimes \mathrm{id}_{\mathrm{g}^{*}}\right)$, followed by a change of variable (the Haar measure on $\mathrm{G}$ corresponds to the Lebesgue measure on $\mathfrak{g}$ under the exponential map). Now, if $f$ does not depend on $x \in \mathrm{G}$, one gets the non-commutative calculus of right-convolution operators on the group; see Remark 4.5.

Clearly there is another strategy, using the expression $X \bullet(-Y)$ in (1.3) and (thus) the expression $\log \left(x y^{-1}\right)$ in (1.4). They provide different (but similar) pseudo-differential calculi, reflecting once again the (eventual) non-commutatativity of our setting. Left convolutions are covered this way.

The global group quantization relies on the formula

$$
\left[\mathrm{Op}_{\mathrm{G} \times \widehat{\mathrm{G}}}(\sigma) u\right](x)=\int_{\mathrm{G}} \int_{\widehat{\mathrm{G}}} \operatorname{Tr}_{\xi}\left[\xi\left(y^{-1} x\right) \sigma(x, \xi)\right] u(y) d \mathrm{~m}(y) d \widehat{\mathrm{m}}(\xi)
$$

involving representation theoretical ingredients. Although the elements of $\widehat{G}$ are, by definition, classes of equivalence, we can treat them simply as irreducible representations chosen each in the corresponding class. We set $d \widehat{\mathrm{m}}(\xi)$ for the Plancherel measure [9,13]. The symbol $\sigma$ can be seen as a family $\{\sigma(x, \xi) \mid(x, \xi) \in$ 
$\mathbf{G} \times \widehat{\mathrm{G}}\}$, where $\sigma(x, \xi)$ is an operator in the Hilbert space $\mathcal{H}_{\xi}$ of the irreducible representation $\xi$. Under various favorable circumstances the ingredients in (1.5) make sense and define an operator acting on various spaces of functions or distributions on G. The theory for graded nilpotent groups, as exposed in [10, 12], is already well developed, but many things can be said [26] even for unimodular type I locally compact groups.

In $\left[26\right.$, Sect. 8] it has been shown that the two quantizations $O p_{G \times \widehat{G}}$ and $O p_{G \times \mathfrak{g}^{*}}$ are actually equivalent in a strong sense, being both obtained from a crossed product $C^{*}$-algebraic construction, by applying two different (but both isomorphic) types of partial Fourier transforms in conjunction with the canonical Schrödinger representation of the crossed product $C^{*}$-algebra. The first Fourier transform is defined in terms of the duality between $G$ and its unitary dual $\widehat{G}$ and relies on the non-commutative Plancherel theorem, valid also for large classes of non nilpotent groups. The second comes from identifying $\mathrm{G}$ with $\mathfrak{g}$ by the exponential map, and then using the duality between $\mathfrak{g}$ and $\mathfrak{g}^{*}$. Composing one partial Fourier transform id $\otimes \mathscr{F}_{(\mathrm{G}, \widehat{G})}$ with the inverse id $\otimes \mathscr{F}_{\left(\mathrm{G}, \mathfrak{g}^{*}\right)}^{-1}$ of the other provides an isomorphism id $\otimes \mathscr{W}$ between the two quantizations; they can be seen as two equivalent ways to represent operators on function spaces over a connected simply connected nilpotent Lie group. Unfortunately, besides being an isomorphism, this composition seems in general rather hard to use being not very explicit.

However, for a subclass of nilpotent Lie groups, a remarkable fact occurs, that is the main subject of the present paper. It is known $[6,22]$ that the unitary dual $\widehat{G}$ can be understood via the coadjoint action of $\mathrm{G}$ on $\mathfrak{g}^{*}$. Actually there is a one-to-one correspondence (homeomorphism) between classes of equivalences of irreducible representations and coadjoint orbits. Moreover, the representation is square integrable with respect to the center if and only if the corresponding coadjoint orbit is flat; we refer to $[6,30]$ and to our Subection 2.2 for these notions. A given nilpotent Lie group might not have any flat coadjoint orbit, but if it does, these types of orbits are generic in a certain sense; in such a case the group and the Lie algebra will be called admissible. There are examples of nilpotent Lie algebras with flat coadjoint orbits of arbitrary large dimension; actually for every $n$ there are $n$-dimensional admissible groups. In addition, every nilpotent Lie algebra can be embedded in such an admissible Lie algebra [6, Ex. 4.5.14].

For these admissible groups we prove our main results, Theorem 3.3 and Corollary 4.2, that need the notion of the Pedersen-Weyl pseudo-differential calculus. This is due to [32] and is briefly exposed in Subsection 3.1. Let us only say that it is a way to tranform functions or distributions defined on any coadjoint orbit $\Omega_{\xi}$ into operators acting on the Hilbert space $\mathcal{H}_{\xi}$ of the irreducible representation $\xi$ corresponding to this orbit. Defining $\operatorname{Ped}_{\xi}$ relies on a special Fourier transform adapted to the coadjoint orbit, involving a predual space $\omega_{\xi} \subset \mathfrak{g}$ of $\Omega_{\xi}$. If flat orbits exist, one can choose the same predual for all of them. Remarkably, if $G$ is the $(2 d+1)$ - dimensional Heisenberg group, Pedersen's quantization (slightly reformulated) is just the Weyl calculus on (generic) $2 d$-dimensional coadjoint obits, that can be identified with the usual phase-space $\mathbb{R}^{2 n}$. It also involves naturally a parameter $\lambda \in \mathbb{R} \backslash\{0\}$, which can actually be understood as a labelling of the orbits.

Returning to the general case, recall our transformation $\mathscr{W}$, sending scalar functions on $\mathfrak{g}^{*}$ into operatorvalued sections on $\widehat{G}$. Assuming that $\mathrm{G}$ is admissible, $\mathscr{W}$ can be described in the following way:

- Pick a Schwartz function $B: \mathfrak{g}^{*} \rightarrow \mathbb{C}$.

- Consider all the restrictions $\left.B_{\xi} \equiv B\right|_{\Omega_{\xi}}$, where $\Omega_{\xi}$ is the coadjoint orbit corresponding to $\xi \in \widehat{G}$.

- Apply the Pedersen quantization of the orbit to get an operator $\operatorname{Ped}_{\xi}\left(B_{\xi}\right)$.

- Then $[\mathscr{W}(B)](\xi)=\operatorname{Ped}_{\xi}\left(B_{\xi}\right)$ for any $\xi \in \widehat{\mathrm{G}}$. 
Consequently, at least within the class of Schwartz symbols, $\mathrm{Op}_{\mathrm{G} \times \widehat{\mathrm{G}}}(\sigma)=\mathrm{Op}_{\mathrm{G} \times \mathfrak{g}^{*}}(f)$ is equivalent to

$$
\sigma(x, \xi)=\operatorname{Ped}_{\xi}\left(\left.f\right|_{\{x\} \times \Omega_{\xi}}\right), \quad \forall x \in \mathrm{G}, \xi \in \widehat{\mathrm{G}} .
$$

This is an extension to admissible groups of a picture that is familiar for the Heisenberg group. The fact that this relation cannot hold for groups without flat coadjoint orbits is explained at the end of Subsection 3.3.

We did not use yet the full power of Kirillov's theory. Besides being operator-valued, the functions or sections on the unitary dual $\widehat{G}$ are hard to study or to use because $\widehat{G}$ itself is, in general, a complicated topological and Borel space, and its Plancherel measure $\widehat{m}$ is also complicated and non-explicit. We describe in Subsection 4.3 how things simplify for admissible groups and how these simplifications are effective at the level of the global group quantization. The emerging concrete picture facilitates a more detailed analytical symbolic calculus, the $\xi \in \widehat{G}$ dependence of the symbols being now replaced by the dependence on a parameter belonging to a (large) open subset $\mathfrak{z}_{\bullet}^{*}$ of the dual $\mathfrak{z}^{*}$ of the center of the Lie algebra. This allows us using Euclidean techniques.

A main tool in a pseudo-differential theory is to define, develop and use a symbolic calculus, in the spirit of the traditional one introduced (among others) by L. Hörmander in the case G $=\mathbb{R}^{n}$. Suitable classes $S_{\rho, \delta}^{m}$ can be found in [10, Sect. 5] for the $O p_{G \times \widehat{G}}$ calculus, if $\mathrm{G}$ is a graded group, and many results involving these spaces of symbols are proven. Their definition makes use of Rockland operators (or sub-Laplacians if G is stratified) and suitable difference operators acting in "the dual variables". By the combination of partial Fourier transforms making the calculi $\mathrm{Op}_{\mathrm{G} \times \widehat{\mathrm{G}}}$ and $\mathrm{Op}_{\mathrm{G} \times \mathfrak{g}^{*}}$ equivalent, one can transfer constructions and results to the $\mathrm{Op}_{\mathrm{G} \times \mathfrak{g}^{*}}$ setting, but this is quite implicit. If, in addition, $\mathrm{G}$ is also admissible, one can use the more transparent (and interesting) form of the transformation $\mathscr{W}$ to get a better understanding. This is done in our Section 5, but the conditions defining $S_{\rho, \delta}^{m}$ - classes on $\mathbf{G} \times \mathfrak{g}^{*}$ still need further investigation.

In Section 6 we do some explicit calculations for a two-step four dimensional admissible graded group. It is shown by direct computations that the relation between $\mathrm{Op}_{\mathrm{G} \times \widehat{\mathrm{G}}}$ and $\mathrm{Op}_{\mathrm{G} \times \mathfrak{g}^{*}}$ holds. The pseudo-differential operators are similar to those appearing for the Heisenberg group; differences appear mainly because now the center of the Heisenberg algebra is 2-dimensional. Computations of the group Fourier transform and the Pedersen quantizations have simple connections with usual Euclidean Fourier transforms and the parametrized Weyl calculus.

By using automorphisms, one can make the link between different corresponding coadjoint orbits or, equivalently, between different corresponding irreducible representations. This can be raised to links between the Pedersen calculi associated to coadjoint orbits that are connected by automorphisms. In particular, if a family of automorphisms acts transitively on the set of flat coadjoint orbits, computations on just one orbit generate easily formulae for all the others. This appears frequently in the literature, in the form of " $\lambda$-Weyl calculi" for the Heisenberg group, where actually $\lambda \in \mathbb{R} \backslash\{0\}$ is a label for the flat coadjoint orbits. We show in an Appendix that this can always be done if the Lie algebra, besides being graded, has a one-dimensional center $\mathfrak{z} \equiv \mathbb{R}$ (a condition not satisfied by our example presented in Section 6). Then the natural dilations associated with the grading, supplemented by an inversion, do act transitively on $\mathfrak{z}_{\bullet}^{*} \equiv \mathbb{R} \backslash\{0\}$ and we are reduced by simple transformations to the case $\lambda=1$. We also present briefly two examples of such Lie algebras, without giving explicitly all the calculations, that can be easily supplied by the reader. They are both graded without being stratified, so, since sub-Laplacians are not available, we indicated in each case a homogeneous positive Rockland operator.

The quantization $O \mathrm{p}_{\mathrm{G} \times \mathrm{g}^{*}}$ for invariant operators has been initiated by Melin [27] and further studied by Głowacki in [17, 18, 19], and the Weyl type quantizations on nilpotent groups have been analysed by a different approach by Manchon [24, 25]. Invariant operators on two-step nilpotent Lie groups have been also 
studied by [29]. Non-invariant pseudo-differential operators on the Heisenberg group have been analysed in [1], with further extensions to graded nilpotent Lie groups in [10, 11].

\section{Framework}

We gather here some notions, notations and conventions, including the coadjoint action, its orbits and their predual vector spaces. Flat coadjoint orbits are discussed in some detail.

\subsection{General facts and coadjoint orbits}

For a given (complex, separable) Hilbert space $\mathcal{H}$, one denotes by $\mathbb{B}(\mathcal{H})$ the $C^{*}$-algebra of all linear bounded operators in $\mathcal{H}$ and by $\mathbb{B}^{2}(\mathcal{H})$ the bi-sided ${ }^{*}$-ideal of all Hilbert-Schmidt operators, which is also a Hilbert space with the scalar product $\langle A, B\rangle_{\mathbb{B}^{2}(\mathcal{H})}:=\operatorname{Tr}\left(A B^{*}\right)$.

Let $\mathrm{G}$ be a connected simply connected nilpotent Lie group with unit e, center $\mathrm{Z}$ and Haar measure $\mathrm{m}$. It is second countable, type I and unimodular. On the unitary dual $\widehat{G}$ of $G$ one has the Mackey Borel structure and the Plancherel measure $\widehat{m}$. Plancherel's Theorem holds [9]. The elements of $\widehat{G}$ are equivalence classes of unitary irreducible strongly continuous Hilbert space representations. In this article we will make a deliberate convenient confusion between classes and elements that represent them.

Let $\mathfrak{g}$ be the Lie algebra of $\mathrm{G}$ with center $\mathfrak{z}=\operatorname{Lie}(Z)$ and $\mathfrak{g}^{*}$ its dual. If $X \in \mathfrak{g}$ and $\mathcal{X} \in \mathfrak{g}^{*}$ we set $\langle X \mid \mathcal{X}\rangle:=\mathcal{X}(X)$. We also denote by $\exp : \mathfrak{g} \rightarrow \mathrm{G}$ the exponential map, which is a diffeomorphism. Its inverse is denoted by $\log : G \rightarrow \mathfrak{g}$. Under these diffeomorphisms the Haar measure on $G$ corresponds to a Haar measure $d X$ on $\mathfrak{g}$ (normalized accordingly). It then follows that $L^{p}(\mathrm{G})$ would be isomorphic to $L^{p}(\mathfrak{g})$. For each $p \in[1, \infty]$, one has an isomorphism

$$
L^{p}(\mathrm{G}) \stackrel{\operatorname{Exp}}{\longrightarrow} L^{p}(\mathfrak{g}), \operatorname{Exp}(u):=u \circ \exp
$$

with inverse

$$
L^{p}(\mathfrak{g}) \stackrel{\log }{\longrightarrow} L^{p}(\mathrm{G}), \log (\nu):=\nu \circ \log .
$$

The Schwartz spaces $\mathcal{S}(\mathrm{G})$ and $\mathcal{S}(\mathfrak{g})$ are defined as in [6, A.2]; they are isomorphic Fréchet spaces.

For $X, Y \in \mathfrak{g}$ we set

$$
X \bullet Y:=\log [\exp (X) \exp (Y)] .
$$

It is a group composition law on $\mathfrak{g}$, given by a polynomial expression in $X, Y$ (the Baker-Campbel-Hausdorff formula). The unit element is 0 and $X^{\bullet} \equiv-X$ is the inverse of $X$ with respect to $\bullet$.

Associated to the (unitary strongly continuous) representation $\xi: G \rightarrow \mathbb{B}\left(\mathcal{H}_{\xi}\right)$, the space of smooth vectors

$$
\mathcal{H}_{\xi}^{\infty}:=\left\{\varphi_{\xi} \in \mathcal{H}_{\xi} \mid \xi(\cdot) \varphi_{\xi} \in C^{\infty}\left(\mathrm{G}, \mathcal{H}_{\xi}\right)\right\}
$$

is a Fréchet space in a natural way and a dense linear subspace of $\mathcal{H}_{\xi}$ which is invariant under the unitary operator $\xi(x)$ for every $x \in \mathrm{G}$. We denote by $\mathcal{H}_{\xi}^{-\infty}$ the space of all continuous antilinear functionals on $\mathcal{H}_{\xi}^{\infty}$ and then we have the natural continuous dense inclusions $\mathcal{H}_{\xi}^{\infty} \hookrightarrow \mathcal{H}_{\xi} \hookrightarrow \mathcal{H}_{\xi}^{-\infty}$.

Now consider the unitary strongly continuous representation $\xi \otimes \bar{\xi}: \mathrm{G} \times \mathrm{G} \rightarrow \mathbb{B}\left[\mathbb{B}^{2}\left(\mathcal{H}_{\xi}\right)\right]$ defined by

$$
(\xi \otimes \bar{\xi})\left(x_{1}, x_{2}\right) T=\xi\left(x_{1}\right) T \xi\left(x_{2}\right)^{-1}, \quad \forall x_{1}, x_{2} \in \mathrm{G}, \forall T \in \mathbb{B}^{2}\left(\mathcal{H}_{\xi}\right) .
$$


The corresponding space of smooth vectors is denoted by $\mathbb{B}\left(\mathcal{H}_{\xi}\right)^{\infty}$ and is called the space of smooth operators for the representation $\xi$. One can prove that $\mathbb{B}\left(\mathcal{H}_{\xi}\right)^{\infty}$ is only formed of trace-class operators. Actually [4, 7] we obtain continuous inclusion maps

$$
\mathbb{B}\left(\mathcal{H}_{\xi}\right)^{\infty} \hookrightarrow \mathbb{B}^{1}\left(\mathcal{H}_{\xi}\right) \hookrightarrow \mathbb{B}\left(\mathcal{H}_{\xi}\right) \simeq\left[\mathbb{B}^{1}\left(\mathcal{H}_{\xi}\right)\right]^{*} \hookrightarrow\left[\mathbb{B}\left(\mathcal{H}_{\xi}\right)^{\infty}\right]^{*}=: \mathbb{B}\left(\mathcal{H}_{\xi}\right)^{-\infty}
$$

The adjoint action $[6,22]$ is

$$
\left.\operatorname{Ad}: G \times \mathfrak{g} \rightarrow \mathfrak{g}, \quad \operatorname{Ad}_{x}(Y):=\left.\frac{d}{d t}\right|_{t=0}\left[x \exp (t Y) x^{-1}\right)\right]
$$

and the coadjoint action of $\mathrm{G}$ is

$$
\operatorname{Ad}^{*}: \mathbf{G} \times \mathfrak{g}^{*} \rightarrow \mathfrak{g}^{*}, \quad(x, \mathcal{Y}) \mapsto \operatorname{Ad}_{x}^{*}(\mathcal{Y}):=\mathcal{Y} \circ \operatorname{Ad}_{x^{-1}}
$$

Denoting by

$$
\text { inn }: \mathrm{G} \times \mathrm{G} \rightarrow \mathrm{G}, \quad(x, y) \mapsto \operatorname{inn}_{x}(y):=x y x^{-1}
$$

the action of $\mathrm{G}$ on itself by inner automorphisms, one has

$$
\operatorname{Ad}_{x}=\log \circ \operatorname{inn}_{x} \circ \exp , \quad \forall x \in \mathrm{G} .
$$

Pick $\mathcal{U} \in \mathfrak{g}^{*}$ with its corresponding coadjoint orbit $\Omega(\mathcal{U}):=\operatorname{Ad}_{\mathrm{G}}^{*}(\mathcal{U}) \subset \mathfrak{g}^{*}$. The isotropy group at $\mathcal{U}$ is

$$
\mathrm{G}_{\mathcal{U}}:=\left\{x \in \mathrm{G} \mid \operatorname{Ad}_{x}^{*}(\mathcal{U})=\mathcal{U}\right\}
$$

with the corresponding isotropy Lie algebra

$$
\mathfrak{g}_{\mathcal{U}}=\operatorname{Lie}\left(\mathrm{G}_{\mathcal{U}}\right)=\left\{X \in \mathfrak{g} \mid \mathcal{U} \circ \operatorname{ad}_{X}=0\right\} \supset \mathfrak{z} .
$$

The coadjoint orbit $\Omega \equiv \Omega(\mathcal{U})$ is a closed submanifold and has a polynomial structure comming from its identification with the symmetric space $G / G_{\mathcal{U}}$. There is a Schwartz space $\mathcal{S}(\Omega)$ and a Poisson algebra structure on $\mathfrak{g}^{*}$ for which the symplectic leaves are exactly the coadjoint orbits. We refer to [22] for details.

Let $n:=\operatorname{dim} \mathfrak{g}$ and fix any sequence of ideals in $\mathfrak{g}$,

$$
\{0\}=\mathfrak{g}_{0} \subset \mathfrak{g}_{1} \subset \cdots \subset \mathfrak{g}_{n}=\mathfrak{g}
$$

such that $\operatorname{dim}\left(\mathfrak{g}_{j} / \mathfrak{g}_{j-1}\right)=1$ and $\left[\mathfrak{g}, \mathfrak{g}_{j}\right] \subset \mathfrak{g}_{j-1}$ for $j=1, \ldots, n$. Pick any $E_{j} \in \mathfrak{g}_{j} \backslash \mathfrak{g}_{j-1}$ for $j=1, \ldots, n$, so that the set $\mathscr{E}:=\left\{E_{1}, \ldots, E_{n}\right\}$ will be a Jordan-Hölder basis in $\mathfrak{g}$. Of course, $\mathfrak{g}_{j}=\operatorname{Span}\left(E_{1}, \ldots, E_{j}\right)$ holds for every $j$. The set of jump indices of the coadjoint orbit $\Omega$ with respect to the above Jordan-Hölder basis is

$$
\epsilon_{\Omega}:=\left\{j \mid \mathfrak{g}_{j} \not \subset \mathfrak{g}_{j-1}+\mathfrak{g}_{\mathcal{U}}\right\}=\left\{j \mid E_{j} \notin \mathfrak{g}_{j-1}+\mathfrak{g}_{\mathcal{U}}\right\}
$$

and does not depend on the choice of $\mathcal{U} \in \Omega$. The corresponding predual of the coadjoint orbit $\Omega$ [32] is

$$
\omega:=\operatorname{Span}\left\{E_{j} \mid j \in \epsilon_{\Omega}\right\} \subset \mathfrak{g}
$$

and it turns out that the map $\left.\Omega \ni \mathcal{Y} \mapsto \mathcal{Y}\right|_{\omega} \in \omega^{*}$ is a diffeomorphism, explaining the terminology. In addition, one has the direct vector sum decomposition $\mathfrak{g}=\mathfrak{g}_{\mathcal{U}} \dot{+} \omega$.

We recall that there is a bijection (even a homeomorphism) between $\widehat{G}$ and the family of all coadjoint orbits; we denote by $\Omega_{\xi}$, with predual $\omega_{\xi}$, the orbit corresponding to the (class of equivalence of the) ireducible representation $\xi: G \rightarrow \mathbb{B}\left(\mathcal{H}_{\xi}\right)$. It is not our intention to review the way this bijection is constructed; see $[6,22]$ for excellent presentations. But we do recall recall, for further use, a concept that is involved in the construction via the theory of induced representations. The Lie subalgebra $\mathfrak{m}$ is polarizing (or maximal subordonate) to the point $\mathcal{U} \in \mathfrak{g}^{*}$ if $\mathcal{U}([\mathfrak{m}, \mathfrak{m}])=0$ and it is maximal with respect to this property. It is known [6, Th. 1.3.3] that for any point there is at least a polarizing algebra. 


\subsection{Flat coadjoint orbits}

A coadjoint orbit $\Omega$ is called flat $[6,30]$ if $\mathfrak{g}_{\mathcal{U}}=\mathfrak{z}$ for some $\mathcal{U} \in \Omega$; then this will also happen for any other element $\mathcal{Y} \in \Omega$ and thus $\mathfrak{g} y$ is an ideal. The flatness condition is equivalent with its corresponding irreducible (class of) representation $\xi$ being square integrable modulo the center and also to the fact that $\Omega=\mathcal{U}+\mathfrak{z}^{\dagger}$ for some $\mathcal{U} \in \mathfrak{g}$ (so it is an affine subspace of $\mathfrak{g}^{*}$ ); we set

$$
\mathfrak{z}^{\dagger}:=\left\{\mathcal{Y} \in \mathfrak{g}^{*}|\mathcal{Y}|_{\mathfrak{z}}=0\right\}
$$

for the annihilator of $\mathfrak{z}$ in the dual. The orbit only depends on the restriction of $\mathcal{U}$ to $\mathfrak{z}$. If such orbits $\Omega$ exist, they are exactly those having maximal dimension.

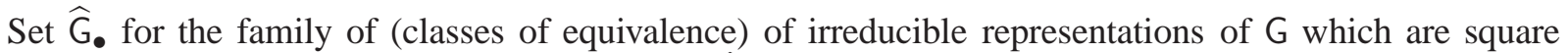
integrable with respect to the center. In many cases $\widehat{G}_{\bullet}$ is void. But in the opposite cases, when flat coadjoint orbits do exist, the Plancherel measure of $\widehat{G}$ is concentrated on $\widehat{G}_{\bullet}$.

Definition 2.1. A connected simply connected nilpotent Lie group $\mathrm{G}$ possessing an irreducible uitary representation which is square integrable modulo the center will be called admissible. Its Lie algebra $\mathfrak{g}$ is also called admissible.

For the full theory of admissible groups we refer to [30] and to [6, Sect. 4.5]. These groups are not necessarily graded [5]. There are criteria for a nilpotent group to have flat coadjoint orbits [6, Prop. 4.5.9].

Remark 2.2. When a flat orbit $\Omega$ exists, we can choose an adapted Jordan-Hölder basis. We set $n:=\operatorname{dim} \mathrm{G}$, $m:=\operatorname{dim} \mathfrak{z}$ and $2 d:=\operatorname{dim} \Omega$; thus one has $n=m+2 d$. Let $\left\{E_{1}, \ldots, E_{m}, E_{m+1}, \ldots, E_{n}\right\}$ be a JordanHölder basis of $\mathfrak{g}$ such that

$$
\mathfrak{z}=\operatorname{Span}\left(E_{1}, \ldots, E_{m}\right)
$$

the jump indices are $\{m+1, \ldots, n\}$. Correspondingly one has

$$
\mathfrak{g}=\mathfrak{z} \oplus \omega
$$

the decomposition is orthogonal with respect to the scalar product on $\mathfrak{g}$ defined by the basis. The same decomposition is obtained for any other flat orbit: there is a common predual for all the flat coadjoint orbits.

In the remaining part of this subsection we are going to summarize here some results from [6, 30]. Let G be an admissible group and choose a Jordan-Hölder basis $\left\{E_{1}, \ldots, E_{m}, E_{m+1}, \ldots, E_{n}\right\}$ of $\mathfrak{g}$ as in Remark 2.2. In terms of annihilators and the dual basis $\left\{\mathcal{E}_{1}, \ldots, \mathcal{E}_{m}, \mathcal{E}_{m+1}, \ldots, \mathcal{E}_{n}\right\}$ in $\mathfrak{g}^{*}$ one has

$$
\operatorname{Span}\left(\mathcal{E}_{1}, \ldots, \mathcal{E}_{m}\right)=\omega^{\dagger} \cong \mathfrak{z}^{*}
$$

and

$$
\operatorname{Span}\left(\mathcal{E}_{m+1}, \ldots, \mathcal{E}_{n}\right)=\mathfrak{z}^{\dagger} \cong \omega^{*}
$$

with

$$
\mathfrak{g}^{*}=\omega^{\dagger} \oplus \mathfrak{z}^{\dagger} \cong \mathfrak{z}^{*} \oplus \omega^{*}
$$

Recall the vector space isomorphisms

$$
\left(\mathfrak{z}^{\dagger}\right)^{\perp}=\left.\omega^{\dagger} \ni \mathcal{X} \rightarrow \mathcal{X}\right|_{\mathfrak{z}} \in \mathfrak{z}^{*} \cong \mathfrak{g}^{*} / \omega^{*} .
$$

Rather often, below, we are going to use the vector space $\mathfrak{z}^{*}$ and some of its subsets; in certain situations a more direct interpretation is through the isomorphic version $\omega^{\dagger}$. 
For any $\mathcal{U} \in \mathfrak{g}^{*}$ we define the skew-symmetric bilinear form

$$
\operatorname{Bil}_{\mathcal{U}}: \mathfrak{g} \times \mathfrak{g} \rightarrow \mathbb{R}, \quad \operatorname{Bil}_{\mathcal{U}}(X, Y):=\langle[X, Y] \mid \mathcal{U}\rangle
$$

If $X \in \mathfrak{z}$ or $Y \in \mathfrak{z}$ one clearly has $\operatorname{Bil}_{\mathcal{U}}(X, Y)=0$. Let us denote by $\operatorname{Bil}_{\mathcal{U}}^{\omega}$ the restriction of $\operatorname{Bil}_{\mathcal{U}}$ to $\omega \times \omega$; it is non-degenerate if and only if the orbit $\Omega(\mathcal{U})$ is flat. Its $\operatorname{Pfaffian} \operatorname{Pf}(\mathcal{U}) \equiv \operatorname{Pf}\left(\operatorname{Bil} \mathcal{U}_{\mathcal{U}}^{\omega}\right) \in \mathbb{R}$ is defined by the relation

$$
\operatorname{Pf}(\mathcal{U})^{2}=\operatorname{det}\left(\operatorname{Bil}_{\mathcal{U}}^{\omega}\right)=\operatorname{det}\left[\left(\mathrm{B}_{\mathcal{U}}^{i j}\right)_{i, j=1, \ldots, 2 d}\right]
$$

where

$$
\mathrm{B}_{\mathcal{U}}^{i, j}:=\operatorname{Bil}_{\mathcal{U}}\left(E_{m+i}, E_{m+j}\right)=\left\langle\left[E_{m+i}, E_{m+j}\right] \mid \mathcal{U}\right\rangle .
$$

The orbit $\Omega(\mathcal{U})=\mathcal{U}+\mathfrak{z}^{\dagger}$ of $\mathcal{U}$ is flat if and only if $\operatorname{Pf}(\mathcal{U}) \neq 0$. It can be checked that $\operatorname{Pf}(\mathcal{U})$ only depends on the restriction of $\mathcal{U}$ to $\mathfrak{z}$, so we get a function

$$
\operatorname{Pf}: \mathfrak{z}^{*} \cong \omega^{\dagger} \rightarrow \mathbb{R}
$$

(a G-invariant homogeneous polynomial in the variable $\mathcal{U} \in \omega^{\dagger}$ ).

Let us set

$$
\mathfrak{g}_{\bullet}^{*}:=\left\{\mathcal{U} \in \mathfrak{g}^{*} \mid \Omega(\mathcal{U}) \text { is flat }\right\}=\left\{\mathcal{U} \in \mathfrak{g}^{*} \mid \operatorname{Pf}(\mathcal{U}) \neq 0\right\} .
$$

The family $\widehat{G}_{\bullet}$ of (classes of equivalence) of irreducible representations of $G$ which are square integrable with respect to the center is endowed with the restriction of the Fell topology on $\widehat{G}$ and with the (full) Plancherel measure. Then Kirillov's homeomorphism $\widehat{G} \cong \mathfrak{g}^{*} / \mathrm{Ad}^{*}$ restricts to

$$
\widehat{G}_{\bullet} \cong \mathfrak{g}_{\bullet}^{*} / \operatorname{Ad}^{*} \text {. }
$$

Using the center $\mathfrak{z}$ of the Lie algebra (or the common predual $\omega$ ) we are going to give a more explicit form of (2.1). The subset

$$
\omega_{\bullet}^{\dagger}:=\omega^{\dagger} \backslash \operatorname{Pf}^{-1}(0)=\mathfrak{g}_{\bullet}^{*} \cap \omega^{\dagger}
$$

or, more conveniently, its isomorphic copy

$$
\mathfrak{z}_{\bullet}^{*}:=\mathfrak{z}^{*} \backslash \operatorname{Pf}^{-1}(0)=\left\{\mathcal{Z} \in \mathfrak{z}^{*} \mid \operatorname{Pf}(\mathcal{Z}) \neq 0\right\},
$$

with the topological and measure-theoretical structures inherited from the vector space of $\mathfrak{z}^{*}$, plays an important role for admissible groups. This is summarised below:

Proposition 2.3. 1. The map

$$
\Xi: \mathfrak{z}_{\bullet}^{*} \rightarrow \widehat{\mathrm{G}}_{\bullet}, \quad \Xi(\mathcal{Z}):=\xi_{\mathcal{Z}+\mathfrak{z}^{\dagger}}
$$

(the equivalence class of irreducible representations associated by Kirillov's theory to the flat coadjoint orbit $\Omega(\mathcal{Z})=\mathcal{Z}+\mathfrak{z}^{\dagger}$ ) is a homeomorhism.

2. The Plancherel measure of $\widehat{\mathrm{G}}$ is concentrated on $\widehat{\mathrm{G}}_{\bullet}$. Transported back through the bijection $\Xi$, it is absolutely continuous with respect to the Lebesgue measure on $\mathfrak{z}_{\bullet}^{*} \subset \mathfrak{z}^{*}$, with density $2^{d} d !|\operatorname{Pf}(\mathcal{Z})|$. 


\section{Fourier transformations and the Weyl-Pedersen calculus}

\subsection{Pedersen quantization of coadjoint orbits}

The arbitrary coadjoint orbit $\Omega \equiv \Omega(\mathcal{U})$ is homeomorphic to the homogeneous space $\mathrm{G} / \mathrm{G}_{\mathcal{U}}$. Since $\mathrm{G}, \mathrm{G}_{\mathcal{U}}$, being both nilpotent, are unimodular, there are $\operatorname{Ad}_{\mathrm{G}}^{*}$-invariant measures on $\Omega$; any two of them are connected by multiplication with a strictly positive constant. We refer to [6, Sect. 4.2, 4.3] for information concerning the normalization of the invariant measure in order to fit with Kirillov's Trace Formula.

Let us recall the Fourier transformation associated to a coadjoint orbit ([32]). For $\Psi \in \mathcal{S}(\Omega)$ we set

$$
\hat{\Psi}: \mathfrak{g} \rightarrow \mathbb{C}, \quad \hat{\Psi}(X):=\int_{\Omega} e^{-i\langle X \mid \mathcal{Y}\rangle} \Psi(\mathcal{Y}) d \gamma_{\Omega}(\mathcal{Y}),
$$

where $\gamma_{\Omega}$ is the canonical invariant measure on $\Omega([6])$. It turns out that $\hat{\Psi} \in C^{\infty}(\mathfrak{g})$ and its restriction to the predual $\omega$ is a Schwartz function. The map

$$
\mathrm{F}_{\Omega}: \mathcal{S}(\Omega) \rightarrow \mathcal{S}(\omega), \quad \mathrm{F}_{\Omega}(\Psi):=\left.\hat{\Psi}\right|_{\omega}
$$

is a linear topological isomorphism called the Fourier transform adapted to the coadjoint orbit $\Omega$. For some (suitably normalized) Lebesgue measure $\lambda_{\omega}$ on $\omega$, its inverse reads

$$
\mathrm{F}_{\Omega}^{-1}: \mathcal{S}(\omega) \rightarrow \mathcal{S}(\Omega), \quad\left[\mathrm{F}_{\Omega}^{-1}(\psi)\right](\mathcal{Y}):=\int_{\omega} e^{i\langle X \mid \mathcal{Y}\rangle} \psi(X) d \lambda_{\omega}(X)
$$

If the coadjoint orbit is associated to $\xi \in \widehat{G}$, we use notations as $\Omega_{\xi}, \omega_{\xi}, \gamma_{\xi}, \lambda_{\xi}$ and $F_{\xi}$. Recall that the predual $\omega_{\xi}$ depends on a Jordan-Hölder basis and that the choice of the invariant measure $\gamma_{\xi}$ fixes $\lambda_{\xi}$ and $F_{\xi}$. Also recall our identification of an irreducible representation with its equivalence class.

If $\psi \in \mathcal{S}\left(\omega_{\xi}\right)$ one sets (in weak sense)

$$
\operatorname{Dep}_{\xi}(\psi):=\int_{\omega_{\xi}} \psi(X) \xi(\exp X) d \lambda_{\xi}(X)
$$

and then, for $\Psi \in \mathcal{S}\left(\Omega_{\xi}\right)$

$$
\operatorname{Ped}_{\xi}(\Psi):=\operatorname{Dep}_{\xi}\left[F_{\xi}(\Psi)\right]=\int_{\omega_{\xi}} \int_{\Omega_{\xi}} e^{-i\langle X \mid \mathcal{X}\rangle} \Psi(\mathcal{X}) \xi(\exp X) d \gamma_{\xi}(\mathcal{X}) d \lambda_{\xi}(X)
$$

We refer to [32] for the properties and the significations of the correspondence $\Psi \mapsto \operatorname{Ped}_{\xi}(\Psi)$ and to [2, 3, 4] for various extensions. In particular, it is known [32] that we get a commuting diagram of linear topological isomorphisms

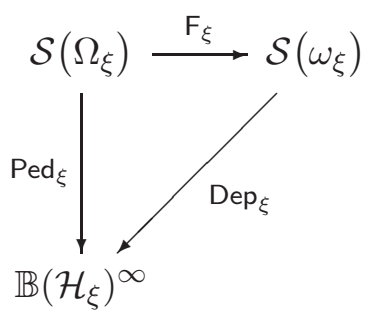


If $\Psi \in \mathcal{S}\left(\Omega_{\xi}\right)$, in terms of the trace $\operatorname{Tr}_{\xi}$ on $B^{1}\left(\mathcal{H}_{\xi}\right)$ one has

$$
\operatorname{Tr}_{\xi}\left[\operatorname{Ped}_{\xi}(\Psi)\right]=\int_{\Omega_{\xi}} \Psi(\mathcal{X}) d \gamma_{\xi}(\mathcal{X})
$$

For the Heisenberg group, by suitable adaptations, one gets in particular the usual Weyl calculus on any of the generic coadjoint orbit. See Subsection 6.4 for anoher explicit example.

Remark 3.1. One also has dequantization formulae as

$$
\operatorname{Dep}_{\xi}^{-1}: \mathbb{B}\left(\mathcal{H}_{\xi}\right)^{\infty} \rightarrow \mathcal{S}\left(\omega_{\xi}\right), \quad\left[\operatorname{Dep}_{\xi}^{-1}(S)\right](X)=\operatorname{Tr}_{\xi}\left[S \xi(\exp X)^{*}\right]
$$

followed by

$$
\begin{gathered}
\operatorname{Ped}_{\xi}^{-1}=\mathrm{F}_{\xi}^{-1} \circ \operatorname{Dep}_{\xi}^{-1}: \mathbb{B}\left(\mathcal{H}_{\xi}\right)^{\infty} \rightarrow \mathcal{S}\left(\Omega_{\xi}\right) \\
{\left[\operatorname{Ped}_{\xi}^{-1}(S)\right](\mathcal{X})=\int_{\omega_{\xi}} e^{i\langle Y \mid \mathcal{X}\rangle} \operatorname{Tr}_{\xi}\left[S \xi(\exp Y)^{*}\right] d \lambda_{\xi}(Y) .}
\end{gathered}
$$

Remark 3.2. N. Pedersen showed in [32, Th 4.1.4.] that $\operatorname{Ped}_{\xi}$ extends to a topological isomorphism $\mathcal{S}^{\prime}\left(\Omega_{\xi}\right) \rightarrow$ $\mathbb{B}\left(\mathcal{H}_{\xi}\right)^{-\infty}$ satifying $\operatorname{Ped}_{\xi}(1)=1_{\xi}$, such that for $\Psi_{1} \in \mathcal{S}\left(\Omega_{\xi}\right), \Psi_{2} \in \mathcal{S}^{\prime}\left(\Omega_{\xi}\right)$ the equality

$$
\left\langle\operatorname{Ped}_{\xi}\left(\Psi_{1}\right), \operatorname{Ped}_{\xi}\left(\Psi_{2}\right)\right\rangle=\Psi_{2}\left(\Psi_{1}\right)
$$

holds in terms of the duality between $\mathbb{B}\left(\mathcal{H}_{\xi}\right)^{\infty}$ and $\mathbb{B}\left(\mathcal{H}_{\xi}\right)^{-\infty}$. If $\operatorname{Ped}_{\xi}\left(\Psi_{2}\right) \in \mathbb{B}\left(\mathcal{H}_{\xi}\right)$ (recall that $\mathbb{B}\left(\mathcal{H}_{\xi}\right)^{\infty} \subset$ $\mathbb{B}^{1}\left(\mathcal{H}_{\xi}\right)$ ) one even has

$$
\operatorname{Tr}_{\xi}\left[\operatorname{Ped}_{\xi}\left(\Psi_{1}\right) \operatorname{Ped}_{\xi}\left(\Psi_{2}\right)\right]=\Psi_{2}\left(\Psi_{1}\right) .
$$

\subsection{Fourier transformations}

Various Fourier integral formulae will be presented below. For the moment $\mathrm{G}$ is connected simply connected and nilpotent; flat coadjoint orbits are not yet needed.

A. There is a Fourier transformation, given by the duality $\left(\mathfrak{g}, \mathfrak{g}^{*}\right)$, defined essentially by

$$
\left(\mathscr{F} \mathfrak{g}_{\mathfrak{g}} \mathfrak{g}^{*} h\right)(\mathcal{X}):=\int_{\mathfrak{g}} e^{-i\langle X \mid \mathcal{X}\rangle} h(X) d X
$$

It is a linear topological isomorphism $\mathscr{F}_{\mathfrak{g}, \mathfrak{g}^{*}}: \mathcal{S}(\mathfrak{g}) \rightarrow \mathcal{S}\left(\mathfrak{g}^{*}\right)$. Using a good normalization of the Lebesgue measure on $\mathfrak{g}^{*}$, it can be seen (after extension) as a unitary map $\mathscr{F}_{\mathfrak{g}, \mathfrak{g}^{*}}: L^{2}(\mathfrak{g}) \rightarrow L^{2}\left(\mathfrak{g}^{*}\right)$.

B. Composing with the isomorphisms Exp and Log one gets Fourier transformations

$$
\begin{gathered}
\mathscr{F}_{\mathrm{G}, \mathfrak{g}^{*}}:=\mathscr{F}_{\mathfrak{g}, \mathfrak{g}^{*}} \circ \operatorname{Exp}: \mathcal{S}(\mathrm{G}) \rightarrow \mathcal{S}\left(\mathfrak{g}^{*}\right), \quad \mathscr{F}_{\mathrm{G}, \mathfrak{g}^{*}}^{-1}:=\log \circ \mathscr{F}_{\mathfrak{g}, \mathfrak{g}^{*}}^{-1}: \mathcal{S}\left(\mathfrak{g}^{*}\right) \rightarrow \mathcal{S}(\mathrm{G}), \\
\left(\mathscr{F}_{\mathrm{G}, \mathfrak{g}^{*}} u\right)(\mathcal{X})=\int_{\mathfrak{g}} e^{-i\langle X \mid \mathcal{X}\rangle} u(\exp X) d X=\int_{\mathrm{G}} e^{-i\langle\log x \mid \mathcal{X}\rangle} u(x) d \mathrm{~m}(x), \\
\left(\mathscr{F}_{\mathrm{G}, \mathfrak{g}^{*}}^{-1} w\right)(x)=\int_{\mathfrak{g}^{*}} e^{i\langle\log x \mid \mathcal{X}\rangle} w(\mathcal{X}) d \mathcal{X} .
\end{gathered}
$$

These maps can also be regarded as unitary isomorphisms of the corresponding $L^{2}$-spaces. 
C. One also has the unitary group Fourier transform

$$
\mathscr{F}_{\mathrm{G}, \widehat{\mathrm{G}}}: L^{2}(\mathrm{G}) \rightarrow \mathscr{B}^{2}(\widehat{\mathrm{G}}):=\int_{\widehat{\mathrm{G}}}^{\oplus} \mathbb{B}^{2}\left(\mathcal{H}_{\xi}\right) d \widehat{\mathrm{m}}(\xi),
$$

defined on $L^{1}(\mathrm{G}) \cap L^{2}(\mathrm{G})$ as

$$
\left(\mathscr{F}_{\mathrm{G}, \widehat{\mathrm{G}}} u\right)(\xi):=\int_{\mathrm{G}} u(x) \xi(x)^{*} d \mathrm{~m}(x),
$$

with inverse (on sufficiently regular elements $b$ )

$$
\left(\mathscr{F}_{\mathrm{G}, \widehat{\mathrm{G}}}^{-1} b\right)(x):=\int_{\widehat{\mathrm{G}}} \operatorname{Tr}_{\xi}[b(\xi) \xi(x)] d \widehat{\mathrm{m}}(\xi) .
$$

It also becomes an isomorphism of Schwartz-type spaces, if we simply define $\mathscr{S}(\widehat{\mathrm{G}})$ to be the image of $\mathcal{S}(\mathrm{G})$ through $\mathscr{F}_{\mathrm{G}, \widehat{\mathrm{G}}}$ with the transported topological structure; the space $\mathscr{S}(\widehat{\mathrm{G}})$ is difficult to describe explicitly (see [16]).

\subsection{The transformation $\mathscr{W}$}

We are now interested in the mapping

$$
\mathscr{W}:=\mathscr{F}_{\mathrm{G}, \widehat{\mathrm{G}}} \circ \mathscr{F}_{\mathrm{G}, \mathfrak{g}^{*}}^{-1}: \mathcal{S}\left(\mathfrak{g}^{*}\right) \rightarrow \mathscr{S}(\widehat{\mathrm{G}})
$$

and its inverse. If $\mathfrak{g}$ is Abelian, identifying $\widehat{G}$ with $\mathfrak{g}^{*}$, it can be seen as the identity mapping. For reasons that will be exposed below (see Remark 3.7 for instance), we will restrict ourselves to admissible groups. As we will see, in this case $\mathscr{W}$ basically consists in restricting the element of $\mathcal{S}\left(\mathfrak{g}^{*}\right)$ to all the coadjoint orbits and then applying the corresponding Pedersen quantizations to these restrictions.

Theorem 3.3. Let $\mathrm{G}$ be an admissible group.

(i) For $B \in \mathcal{S}\left(\mathfrak{g}^{*}\right)$ and $\xi \in \widehat{\mathrm{G}}$ set

$$
B_{\xi}:=\left.B\right|_{\Omega_{\xi}} \text { and } \quad b(\xi):=\operatorname{Ped}_{\xi}\left(B_{\xi}\right) .
$$

Then $b(\xi) \in \mathbb{B}\left(\mathcal{H}_{\xi}\right)^{\infty}$ and one has

$$
b=\mathscr{W}(B) \in \mathscr{S}(\widehat{\mathrm{G}}) .
$$

(ii) Conversely, let

$$
b \equiv\{b(\xi) \mid \xi \in \widehat{\mathrm{G}}\} \in \mathscr{S}(\widehat{\mathrm{G}}) .
$$

For every $\xi \in \widehat{\mathrm{G}}$ and every $\mathcal{X} \in \Omega_{\xi}$ one has

$$
\left[\mathscr{W}^{-1}(b)\right](\mathcal{X})=\int_{\omega_{\xi}} e^{i\langle Y \mid \mathcal{X}\rangle} \operatorname{Tr}_{\xi}\left[b(\xi) \xi(\exp Y)^{*}\right] d \lambda_{\xi}(Y) .
$$

We are going to need two lemmas. The first one gives a first (rather weak) control on the map $\xi \mapsto$ $\operatorname{Ped}_{\xi}\left(B_{\xi}\right)$. The direct integral Banach space $\mathscr{B}^{1}(\widehat{\mathrm{G}})$ is defined similarly to $\mathscr{B}^{2}(\widehat{\mathrm{G}})$, but with respect to the norm

$$
\|\phi\|_{\mathscr{B}^{1}(\widehat{\mathrm{G}})}:=\int_{\widehat{\mathrm{G}}}\|\phi(\xi)\|_{\mathbb{B}^{1}\left(\mathcal{H}_{\xi}\right)} d \widehat{\mathrm{m}}(\xi) .
$$


Lemma 3.4. For any $B \in \mathcal{D}\left(\mathfrak{g}^{*}\right) \equiv C_{\mathrm{c}}^{\infty}\left(\mathfrak{g}^{*}\right)$ one has $b(\cdot) \in \mathscr{B}^{1}(\widehat{\mathrm{G}}) \cap \mathscr{B}^{2}(\widehat{\mathrm{G}})$.

Proof. Set $K:=\operatorname{supp}(B)$ (a compact subset of $\left.\mathfrak{g}^{*}\right)$. Recall that $\widehat{\mathrm{G}}$ is homeomorphic to the quotient of $\mathfrak{g}^{*}$ by the coadjoint action: one has $\mathfrak{g}^{*} \stackrel{q}{\longrightarrow} \mathfrak{g}^{*} / \mathrm{Ad}^{*} \cong \widehat{\mathrm{G}}$. Then for $\Omega_{\xi}$ not belonging to the (quasi-)compact subset $K^{\prime}:=q(K)$ of $\mathfrak{g}^{*} /$ Ad $^{*}$, meaning that $\Omega_{\xi} \cap K=\emptyset$, one has $\left.B\right|_{\Omega_{\xi}}=0$. Thus $b(\xi)=0$ if $\xi$ belongs to the complement of the homeomorphic compact image of $K^{\prime}$ in $\widehat{\mathrm{G}}$.

The Plancherel measure is bounded on compact subsets [9, 18.8.4], so one gets $b \in \mathscr{B}^{1}(\widehat{\mathrm{G}}) \cap \mathscr{B}^{2}(\widehat{\mathrm{G}})$ if $\xi \mapsto \operatorname{Ped}_{\xi}\left(B_{\xi}\right)$ is essentially locally bounded. Since the Plancherel measure is concentrated on the set of generic flat orbits, only the corresponding irreducible representations $\xi$ are important. Then local boundedness follows easily from [3, Th. 4.4] and the fact that $B \in \mathcal{D}\left(\mathfrak{g}^{*}\right)$.

Lemma 3.5. For any $C \in \mathcal{S}\left(\mathfrak{g}^{*}\right)$ one has

$$
\int_{\widehat{\mathrm{G}}}\left[\left.\int_{\Omega_{\xi}} C\right|_{\Omega_{\xi}}(\mathcal{X}) d \gamma_{\xi}(\mathcal{X})\right] d \widehat{\mathrm{m}}(\xi)=\int_{\mathfrak{g}^{*}} C(\mathcal{X}) d \mathcal{X} .
$$

Proof. See [6, Pag. 153-154] or [22, Page 100].

One can now prove Theorem 3.3.

Proof. (i) Taking into account the way $\mathscr{W}$ is defined, the identity $b=\mathscr{W}(B)$ is equivalent to

$$
\mathscr{F}_{\mathrm{G}, \widehat{\mathrm{G}}}^{-1}(b)=\mathscr{F}_{\mathrm{G}, \mathfrak{g}^{*}}^{-1}(B) \text {. }
$$

It is enough to assume $B \in \mathcal{D}\left(\mathfrak{g}^{*}\right)$; then clearly $B_{\xi} \in \mathcal{D}\left(\Omega_{\xi}\right) \subset \mathcal{S}\left(\Omega_{\xi}\right)$ and thus

$$
b(\xi) \in \mathbb{B}\left(\mathcal{H}_{\xi}\right)^{\infty} \subset \mathbb{B}^{1}\left(\mathcal{H}_{\xi}\right) \subset \mathbb{B}^{2}\left(\mathcal{H}_{\xi}\right) .
$$

In the computation below we will need to apply formula (3.3) as it is (pointwise). Recall (cf. [15]) that $\mathscr{F}_{\mathrm{G}, \widehat{G}}$ restricts to an isomorphism

$$
L^{1}(\mathrm{G}) \cap A(\mathrm{G}) \rightarrow \mathscr{B}^{1}(\widehat{\mathrm{G}}) \cap \mathscr{B}^{2}(\widehat{\mathrm{G}}),
$$

where $A(\mathrm{G})$ is Eymard's Fourier algebra. In addition, if $\phi \in \mathscr{B}^{1}(\widehat{\mathrm{G}}) \cap \mathscr{B}^{2}(\widehat{\mathrm{G}})$, the inversion formula (3.3) holds pointwisely. But it is shown in Lemma 3.4 that $b(\cdot) \in \mathscr{B}^{1}(\widehat{\mathrm{G}}) \cap \mathscr{B}^{2}(\widehat{\mathrm{G}})$, so we have pointwisely

$$
\left[\mathscr{F}_{\mathrm{G}, \widehat{\mathrm{G}}}^{-1}(b)\right](x)=\int_{\widehat{\mathrm{G}}} \operatorname{Tr}_{\xi}[b(\xi) \xi(x)] d \widehat{\mathrm{m}}(\xi)
$$

We work with $x=\exp X$; by Remark 3.2, there is a unique distribution $\Phi_{X}^{(\xi)} \in \mathcal{S}^{\prime}\left(\Omega_{\xi}\right)$ such that

$$
\xi(\exp X)=\operatorname{Ped}_{\xi}\left(\Phi_{X}^{(\xi)}\right)
$$

with

$$
\operatorname{Tr}_{\xi}[b(\xi) \xi(\exp X)]=\operatorname{Tr}_{\xi}\left[\operatorname{Ped}_{\xi}\left(B_{\xi}\right) \operatorname{Ped}_{\xi}\left(\Phi_{X}^{(\xi)}\right)\right]=\Phi_{X}^{(\xi)}\left(B_{\xi}\right) .
$$

Computing the Pedersen symbol $\Phi_{X}^{(\xi)}$ of $\xi(\exp X)$ in general seems to be difficult. But if $\mathrm{G}$ is admissible, since $\widehat{G} \backslash \widehat{G}$. is $\widehat{m}$-negligible, in (3.7) we can concentrate on flat orbits and use a result from [32].

Assuming that $\Omega_{\xi}=\mathcal{U}+\mathfrak{z}^{\dagger}$ is flat, let us decompose

$$
X=X_{\mathfrak{z}}+X_{\omega} \in \mathfrak{z} \oplus \omega=\mathfrak{g}
$$


(see Remark 2.2). Note that $\exp X=\exp X_{\mathfrak{z}} \exp X_{\omega}$; higher order terms in the BCH formula are trivial, since $X_{\mathfrak{z}}$ is central. The central character $\chi_{\xi}: Z \rightarrow \mathbb{T}$ of the irreducible representation $\xi$ is defined by

$$
\xi(z)=\chi_{\xi}(z) 1_{\xi}, \quad \forall z \in \mathbf{Z}
$$

and is given by $\chi_{\xi}(z)=e^{i\langle\log z \mid \mathcal{U}\rangle}$ (independent on the choice of the point $\mathcal{U} \in \Omega_{\xi}$ ); thus we have

$$
\xi(\exp X)=\xi\left(\exp X_{\mathfrak{z}}\right) \xi\left(\exp X_{\omega}\right)=e^{i\left\langle X_{\mathfrak{z}} \mid \mathcal{U}\right\rangle} \xi\left(\exp X_{\omega}\right)
$$

Then (some steps will be explained below)

$$
\begin{aligned}
\operatorname{Tr}_{\xi}\left[\operatorname{Ped}_{\xi}\left(B_{\xi}\right) \xi(\exp X)\right] & =e^{i\left\langle X_{\mathfrak{z}} \mid \mathcal{U}\right\rangle} \operatorname{Tr}_{\xi}\left[\operatorname{Dep}_{\xi}\left(\mathrm{F}_{\xi}\left(B_{\xi}\right)\right) \xi\left(\exp X_{\omega}\right)\right] \\
& =e^{i\left\langle X_{\mathfrak{z}} \mid \mathcal{U}\right\rangle}\left(\mathrm{F}_{\xi}\left(B_{\xi}\right)\right)\left(-X_{\omega}\right) \\
& =e^{i\left\langle X_{\mathfrak{z}} \mid \mathcal{U}\right\rangle} \int_{\Omega_{\xi}} e^{i\left\langle X_{\omega} \mid \mathcal{X}\right\rangle} B_{\xi}(\mathcal{X}) d \gamma_{\xi}(\mathcal{X}) \\
& =\int_{\Omega_{\xi}} e^{i\langle X \mid \mathcal{X}\rangle} B_{\xi}(\mathcal{X}) d \gamma_{\xi}(\mathcal{X}) .
\end{aligned}
$$

The second equality is equivalent to Lemma 4.1.2 from [32], relying on the deep result [32, Th. 2.1.1]; note that $\operatorname{Dep}_{\xi}$ corresponds to the notation $T$ of Pedersen, and the extra constant in [32, Lemma 4.1.2] is due to different conventions. For the last equality recall that $\Omega_{\xi}=\mathcal{U}+\mathfrak{z}^{\perp}$, which allows one to write for each $\mathcal{X} \in \Omega_{\xi}$

$$
\left\langle X_{\mathfrak{z}} \mid \mathcal{U}\right\rangle+\left\langle X_{\omega} \mid \mathcal{X}\right\rangle=\left\langle X_{\mathfrak{z}} \mid \mathcal{X}\right\rangle+\left\langle X_{\omega} \mid \mathcal{X}\right\rangle=\langle X \mid \mathcal{X}\rangle .
$$

Replacing this above and also recalling (3.6) and (3.2), one gets

$$
\begin{aligned}
{\left[\mathscr{F}_{\mathrm{G}, \widehat{\mathrm{G}}}^{-1}(b)\right](\exp X) } & =\int_{\widehat{\mathrm{G}}}\left[\int_{\Omega_{\xi}} e^{i\langle X \mid \mathcal{X}\rangle} B_{\xi}(\mathcal{X}) d \gamma_{\xi}(\mathcal{X})\right] d \widehat{\mathrm{m}}(\xi) \\
& =\int_{\mathfrak{g}^{*}} e^{i\langle X \mid \mathcal{X}\rangle} B(\mathcal{X}) d \mathcal{X}=\left[\mathscr{F}_{\mathrm{G}, \mathfrak{g}^{*}}^{-1}(B)\right](\exp X) .
\end{aligned}
$$

Now, once the identity $b=\mathscr{W}(B)$ is proven, the fact that $b \in \mathscr{S}(\widehat{\mathrm{G}})$ is clear from $\mathscr{S}(\widehat{\mathrm{G}}):=\mathscr{F}_{\mathrm{G}, \widehat{\mathrm{G}}}[\mathcal{S}(\mathrm{G})]$ and the definition of $\mathscr{W}$.

(ii) To compute $\mathscr{W}^{-1}$, seen as the inverse of $\mathscr{W}$ already made explicit at point 1 , we have to use the dequantisation formulae of Remark 3.1: First recall that $b \in \mathscr{S}(\widehat{\mathrm{G}})$ means by definition that

$$
b=\mathscr{F}_{\mathrm{G}, \widehat{\mathrm{G}}}(c) \text {, with } c \in \mathcal{S}(\mathrm{G}) \text {. }
$$

In [21, Th. 3.4] it is shown that for every $\xi$ the map $c \mapsto\left[\mathscr{F}_{\mathrm{G}, \widehat{\mathrm{G}}}(c)\right](\xi)$ sends $\mathcal{S}(\mathrm{G})$ (surjectively but not injectively) to $\mathbb{B}\left(\mathcal{H}_{\xi}\right)^{\infty}$. Therefore $b(\xi)$ belongs to $\mathbb{B}\left(\mathcal{H}_{\xi}\right)^{\infty}$ and we can construct

$$
B_{\xi}:=\operatorname{Ped}_{\xi}^{-1}[b(\xi)] \in \mathcal{S}\left(\Omega_{\xi}\right)
$$

given by (3.1). For $\mathcal{X} \in \mathfrak{g}^{*}$ we put $B(\mathcal{X}):=B_{\xi}(\mathcal{X})$, selecting $\xi$ such that $\mathcal{X} \in \Omega_{\xi}$. Then (3.1) leads finally to the formula (3.5) for $B=\mathscr{W}^{-1}(b)$.

Let us briefly indicate how a weaker form of the point (i) of Theorem 3.3 follows from a result in [32]. 
Proof. Using our notations, Theorem 4.2.1 in [32] states that for every $u \in \mathcal{S}(\mathrm{G})$ and every $\xi \in \widehat{\mathrm{G}}$ which is square integrable modulo the center, one has

$$
\left[\mathscr{F}_{\mathrm{G}, \widehat{\mathrm{G}}}(u)\right](\xi)=\operatorname{Ped}_{\xi}\left[\left.\mathscr{F}_{\mathfrak{g}, \mathfrak{g}^{*}}(u \circ \exp )\right|_{\Omega_{\xi}}\right] .
$$

Setting $u:=\left[\mathscr{F}_{\mathfrak{g}, \mathfrak{g}^{*}}^{-1}(B)\right] \circ \log =\mathscr{F}_{\mathrm{G}, \mathfrak{g}^{*}}^{-1}(B)$ we get for such $\xi$

$$
[\mathscr{W}(B)](\xi):=\mathscr{F}_{\mathrm{G}, \widehat{\mathrm{G}}}\left[\mathscr{F}_{\mathrm{G}, \mathfrak{g}^{*}}^{-1}(B)\right](\xi)=\operatorname{Ped}_{\xi}\left(\left.B\right|_{\Omega_{\xi}}\right)=: b(\xi) .
$$

Thus we checked that $\mathscr{W}(B)$ and $b$ defined in Theorem 3.3 coincide on the set of square integrable modulo the center irreducible representations. However, it seems rather hard to go further. Neither regularity properties of the implicitly defined space $\mathscr{S}(\widehat{\mathrm{G}})$, nor the smoothness of $b$ are obvious a priori.

Corollary 3.6. Let $\xi$ be an irreducible representation of the admissible group $\mathrm{G}$, that is square integrable modulo the center, and let

$$
\widetilde{\xi}(v):=\int_{\mathrm{G}} v(x) \xi(x) d \mathrm{~m}(x), \quad v \in \mathcal{S}(\mathrm{G}),
$$

its integrated form acting on the Schwartz space. One has

$$
\operatorname{ker}(\widetilde{\xi})=\left\{v \in \mathcal{S}(\mathrm{G})\left|\left[\mathscr{F}_{\mathrm{G}, \mathfrak{g}^{*}}(v)\right]\right|_{\Omega_{\xi}}=0\right\}
$$

Proof. Let $v:=\mathscr{F}_{\mathrm{G}, \mathfrak{g}^{*}}^{-1}(B) \in \mathcal{S}(\mathrm{G})$ with $B=\mathscr{F}_{\mathrm{G}, \mathfrak{g}^{*}}(v) \in \mathcal{S}\left(\mathfrak{g}^{*}\right)$ and set as above $b(\eta):=\operatorname{Ped}_{\eta}\left(\left.B\right|_{\Omega_{\eta}}\right)$ for every $\eta \in \widehat{G}$. With these notations, and using our result (3.4), one has

$$
\widetilde{\xi}(v)=\left(\mathscr{F}_{\mathrm{G}, \widehat{\mathrm{G}}}\left[\mathscr{F}_{\mathrm{G}, \mathfrak{g}^{*}}^{-1}(B)\right]\right)(\xi)=b(\xi) .
$$

Since the map Ped $\operatorname{Pis}_{\xi}$ is isomorphism, one has $\widetilde{\xi}(v)=0$ if and only if $b(\xi)=0$ and if and only if $\left.B\right|_{\Omega_{\xi}}=$ $\left.\mathscr{F}_{\mathrm{G}, \mathfrak{g}^{*}}^{\Phi}(v)\right|_{\Omega_{\xi}}=0$.

Remark 3.7. Working with a general connected simply connected nilpotent Lie group G, Ludwig [23] defines a (two-sided self-adjoint) ideal $\mathcal{J}$ of $L^{1}(\mathrm{G})$ to be $\operatorname{good}$ if $\operatorname{Exp}(\mathcal{J})$ is an ideal in $L^{1}(\mathfrak{g})$ with the obvious convolution multiplication. Let

$$
\widetilde{\xi}: \mathcal{S}(\mathrm{G}) \rightarrow \mathbb{B}\left(\mathcal{H}_{\xi}\right)^{\infty}, \quad \widetilde{\xi}(v):=\left[\mathscr{F}_{\mathrm{G}, \widehat{\mathrm{G}}}(v)\right](\xi)=\int_{\mathrm{G}} v(x) \xi(x)^{*} d \mathrm{~m}(x)
$$

the integrated form of $\xi \in \widehat{G}$. Then, for an element $\xi$ of $\widehat{G}$, he shows that $\operatorname{ker} \widetilde{\xi}$ is good if and only if $\Omega_{\xi}$ is an affine subspace and if and only if

$$
\operatorname{ker}(\widetilde{\xi})=\left\{v \in L^{1}(\mathrm{G})\left|\mathscr{F}_{\mathrm{G}, \mathfrak{g}^{*}}(v)\right|_{\Omega_{\xi}}=0\right\} .
$$

It is easy to see that this forbids Theorem 3.3 to hold for non-admissible groups. This has its roots in the form of the Pedersen symbol $\Phi_{X}^{(\xi)}$ of $\xi(\exp X)$ for general $\xi$ and it is probably related to the necessity of introducing a modified Fourier transformation instead of $\mathscr{F}_{\mathrm{G}, \mathfrak{g}^{*}}$.

\section{Various quantizations and their mutual connections}

In this section we discuss different quantizations on $\mathrm{G}$ and relations among them. 


\subsection{A list of quantizations}

One has various quantizations on the "phase spaces" $\mathfrak{g} \times \mathfrak{g}^{*} \ni(X, \mathcal{X}), \mathrm{G} \times \mathfrak{g}^{*} \ni(x, \mathcal{X}), \mathrm{G} \times \widehat{\mathrm{G}} \ni(x, \xi)$ :

$$
\begin{aligned}
& \mathfrak{O p}_{\mathfrak{g} \times \mathfrak{g}^{*}}: L^{2}\left(\mathfrak{g} \times \mathfrak{g}^{*}\right) \rightarrow \mathbb{B}^{2}\left[L^{2}(\mathfrak{g})\right], \\
& {\left[\mathfrak{O} \mathfrak{p}_{\mathfrak{g} \times \mathfrak{g}^{*}}(\mathfrak{f}) \nu\right](X)=\int_{\mathfrak{g}} \int_{\mathfrak{g}^{*}} e^{i\langle(-Y) \bullet X \mid \mathcal{X}\rangle} \mathfrak{f}(X, \mathcal{X}) \nu(Y) d Y d \mathcal{X}}
\end{aligned}
$$

and

$$
\begin{aligned}
& O p_{\mathfrak{g} \times \mathfrak{g}^{*}}: L^{2}\left(\mathfrak{g} \times \mathfrak{g}^{*}\right) \rightarrow \mathbb{B}^{2}\left[L^{2}(\mathrm{G})\right], \quad O \mathfrak{p}_{\mathfrak{g} \times \mathfrak{g}^{*}}(\mathrm{f})=\operatorname{Exp} \circ \mathfrak{O p}_{\mathfrak{g} \times \mathfrak{g}^{*}}(\mathrm{f}) \circ \log , \\
& {\left[O \mathrm{p}_{\mathfrak{g} \times \mathfrak{g}^{*}}(\mathrm{f}) u\right](x)=\int_{\mathbf{G}} \int_{\mathfrak{g}^{*}} e^{i\left\langle\log \left(y^{-1} x\right) \mid \mathcal{X}\right\rangle} \mathrm{f}(\log x, \mathcal{X}) u(y) d \mathbf{m}(y) d \mathcal{X}}
\end{aligned}
$$

and the one we prefer

$$
\begin{aligned}
& O \mathrm{p}_{\mathrm{G} \times \mathfrak{g}^{*}}:=O \mathrm{Op}_{\mathfrak{g} \times \mathfrak{g}^{*}} \circ(\operatorname{Exp} \otimes \mathrm{id}): L^{2}\left(\mathrm{G} \times \mathfrak{g}^{*}\right) \rightarrow \mathbb{B}^{2}\left[L^{2}(\mathrm{G})\right], \\
& {\left[\mathrm{Op}_{\mathrm{G} \times \mathfrak{g}^{*}}(f) u\right](x)=\int_{\mathrm{G}} \int_{\mathfrak{g}^{*}} e^{i\left\langle\log \left(y^{-1} x\right) \mid \mathcal{X}\right\rangle} f(x, \mathcal{X}) u(y) d \mathrm{~m}(y) d \mathcal{X} .}
\end{aligned}
$$

Remark 4.1. Taking into account Schwartz's Kernel Theorem and the way various Schwartz spaces were defined, one gets topological linear isomorphisms

$$
\begin{array}{ll}
O p_{\mathfrak{g} \times \mathfrak{g}^{*}}: \mathcal{S}\left(\mathfrak{g} \times \mathfrak{g}^{*}\right) \stackrel{\sim}{\longrightarrow} \mathbb{B}^{2}\left[\mathcal{S}^{\prime}(\mathrm{G}), \mathcal{S}(\mathrm{G})\right], & O p_{\mathfrak{g} \times \mathfrak{g}^{*}}: \mathcal{S}^{\prime}\left(\mathfrak{g} \times \mathfrak{g}^{*}\right) \stackrel{\sim}{\longrightarrow} \mathbb{B}^{2}\left[\mathcal{S}(\mathrm{G}), \mathcal{S}^{\prime}(\mathrm{G})\right] \\
\mathrm{Op}_{\mathrm{G} \times \mathfrak{g}^{*}}: \mathcal{S}\left(\mathrm{G} \times \mathfrak{g}^{*}\right) \stackrel{\sim}{\longrightarrow} \mathbb{B}^{2}\left[\mathcal{S}^{\prime}(\mathrm{G}), \mathcal{S}(\mathrm{G})\right], & \mathrm{Op}_{\mathrm{G} \times \mathfrak{g}^{*}}: \mathcal{S}^{\prime}\left(\mathrm{G} \times \mathfrak{g}^{*}\right) \stackrel{\sim}{\longrightarrow} \mathbb{B}^{2}\left[\mathcal{S}(\mathrm{G}), \mathcal{S}^{\prime}(\mathrm{G})\right] .
\end{array}
$$

Finally, recall that we were interested in the global group quantization [10,26] (irreducible representations are still identified to corresponding equivalence classes)

$$
\begin{array}{r}
\mathrm{Op}_{\mathrm{G} \times \widehat{\mathrm{G}}}: \mathscr{B}^{2}(\mathrm{G} \times \widehat{\mathrm{G}}):=L^{2}(\mathrm{G}) \otimes \mathscr{B}^{2}(\widehat{\mathrm{G}}) \rightarrow \mathbb{B}^{2}\left[L^{2}(\mathrm{G})\right], \\
{\left[\mathrm{Op}_{\mathrm{G} \times \widehat{\mathrm{G}}}(\sigma) u\right](x)=\int_{\mathrm{G}} \int_{\widehat{\mathrm{G}}} \operatorname{Tr}_{\xi}\left[\xi\left(y^{-1} x\right) \sigma(x, \xi)\right] u(y) d \mathrm{~m}(y) d \widehat{\mathrm{m}}(\xi) .}
\end{array}
$$

\subsection{Connections among quantizations}

It has been shown in [26] that $O p_{G \times \widehat{G}}$ and $O p_{G \times g^{*}}$ are also equivalent, for general connected simply connected nilpotent Lie groups. Actually one has the following commutative diagram of isomorphisms:

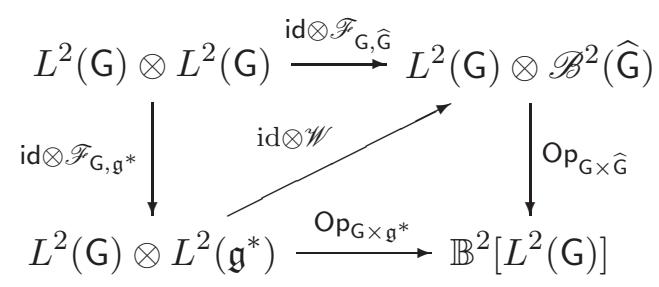

The reason is that one can write

$$
\mathrm{Op}_{\mathrm{G} \times \widehat{\mathrm{G}}}=\operatorname{Int} \circ \mathrm{CV} \circ\left(\mathrm{id} \otimes \mathscr{F}_{\mathrm{G}, \widehat{\mathrm{G}}}^{-1}\right) \quad \text { and } \quad \mathrm{Op}_{\mathrm{G} \times \mathfrak{g}^{*}}=\operatorname{Int} \circ \mathrm{CV} \circ\left(\mathrm{id} \otimes \mathscr{F}_{\mathrm{G}, \mathfrak{g}^{*}}^{-1}\right)
$$


where, besides the Fourier transformations already introduced, $\operatorname{lnt}(K)$ is the integral operator of kernel $K$ and $\mathrm{CV}$ means composition with the change of variables

$$
\mathrm{cv}: \mathrm{G} \times \mathrm{G} \rightarrow \mathrm{G} \times \mathrm{G}, \quad \operatorname{cv}(x, y):=\left(x, y^{-1} x\right) .
$$

Using the isomorphism $\mathscr{W}$, for $A \in L^{2}(\mathrm{G}) \otimes L^{2}\left(\mathfrak{g}^{*}\right)$ one has

$$
\mathrm{Op}_{\mathrm{G} \times \widehat{\mathrm{G}}}[(\mathrm{id} \otimes \mathscr{W}) f]=\mathrm{Op}_{\mathrm{G} \times \mathfrak{g}^{*}}(f) .
$$

This is quite easy to handle for admissible groups, since $\mathscr{W}$ has the simple form given by Theorem 3.3.

Corollary 4.2. Assume that $\mathrm{G}$ is an admissible group. For $f \in \mathcal{S}\left(\mathrm{G} \times \mathfrak{g}^{*}\right)$ and $(x, \xi) \in \mathrm{G} \times \widehat{\mathrm{G}}$, let us set $f_{(x, \xi)} \in \mathcal{S}\left(\Omega_{\xi}\right)$ through

$$
f_{(x, \xi)}(\mathcal{X}):=f(x, \mathcal{X}) \text { for every } \mathcal{X} \in \Omega_{\xi},
$$

i.e. $f_{(x, \xi)}$ is the restriction of $f$ to the subset $\{x\} \times \Omega_{\xi}$, seen as a function $\Omega_{\xi} \rightarrow \mathbb{C}$. The expression

$$
\sigma(x, \xi):=\operatorname{Ped}_{\xi}\left(f_{(x, \xi)}\right)=\int_{\omega_{\xi}}\left[F_{\xi}\left(f_{(x, \xi)}\right)\right](Y) \xi(\exp Y) d \lambda_{\xi}(Y) \in \mathbb{B}\left(\mathcal{H}_{\xi}\right)^{\infty}
$$

denotes the Pedersen quantization of this symbol, associated to the coadjoint orbit $\Omega_{\xi}$. Then one has

$$
(\mathrm{id} \otimes \mathscr{W})(f)=\sigma \quad \text { and } \quad \mathrm{Op}_{\mathrm{G} \times \widehat{\mathrm{G}}}(\sigma)=\mathrm{Op}_{\mathrm{G} \times \mathfrak{g}^{*}}(f) .
$$

Proof. This follows from Theorem 3.3 and from (4.5).

Remark 4.3. We described the correspondence $\mathcal{S}\left(\mathrm{G} \times \mathfrak{g}^{*}\right) \ni f \mapsto \sigma \in \mathscr{S}(\mathrm{G} \times \widehat{\mathrm{G}})$. For the reverse one, we have to use the dequantization formulae of Remark 3.1: Suppose we are given

$$
\sigma \equiv\left\{\sigma(x, \xi) \in \mathbb{B}\left(\mathcal{H}_{\xi}\right)^{\infty} \mid(x, \xi) \in \mathrm{G} \times \widehat{\mathrm{G}}\right\} \in \mathscr{S}(\mathrm{G} \times \widehat{\mathrm{G}}) .
$$

For each $(x, \xi)$ we construct

$$
f_{(x, \xi)}:=\operatorname{Ped}_{\xi}^{-1}[\sigma(x, \xi)] \in \mathcal{S}\left(\Omega_{\xi}\right)
$$

given by (3.1) and then, for $(x, \mathcal{X}) \in \mathrm{G} \times \mathfrak{g}^{*}$ with $\mathcal{X} \in \Omega_{\xi}$, we put

$$
f(x, \mathcal{X}):=f_{(x, \xi)}(\mathcal{X}) .
$$

This leads finally to the formula

$$
f(x, \mathcal{X})=\int_{\omega_{\xi}} e^{i\langle Y \mid \mathcal{X}\rangle} \operatorname{Tr}_{\xi}\left[\sigma(x, \xi) \xi(\exp Y)^{*}\right] d \lambda_{\xi}(Y), \quad \forall(x, \mathcal{X}) \in \mathrm{G} \times \Omega_{\xi} .
$$

Remark 4.4. Obviously one can introduce composition laws

$$
\begin{aligned}
\mathcal{S}\left(\Omega_{\xi}\right) \times \mathcal{S}\left(\Omega_{\xi}\right) \stackrel{\sharp_{\xi}}{\longrightarrow} \mathcal{S}\left(\Omega_{\xi}\right), & \operatorname{Ped}_{\xi}\left(\Psi_{1} \sharp_{\xi} \Psi_{2}\right):=\operatorname{Ped}_{\xi}\left(\Psi_{1}\right) \operatorname{Ped}_{\xi}\left(\Psi_{2}\right), \\
\mathscr{S}(\mathrm{G} \times \widehat{\mathrm{G}}) \times \mathscr{S}(\mathrm{G} \times \widehat{\mathrm{G}}) \stackrel{\#_{\mathrm{G} \times \widehat{\mathrm{G}}}}{\longrightarrow} \mathscr{S}(\mathrm{G} \times \widehat{\mathrm{G}}), & \operatorname{Op}_{\mathrm{G} \times \widehat{\mathrm{G}}}\left(\sigma_{1} \#{ }_{\mathrm{G} \times \widehat{\mathrm{G}}} \sigma_{2}\right):=\operatorname{Op}_{\mathrm{G} \times \widehat{\mathrm{G}}}\left(\sigma_{1}\right) \operatorname{Op}_{\mathrm{G} \times \widehat{\mathrm{G}}}\left(\sigma_{2}\right), \\
\mathcal{S}\left(\mathrm{G} \times \mathfrak{g}^{*}\right) \times \mathcal{S}\left(\mathrm{G} \times \mathfrak{g}^{*}\right) \stackrel{\#_{\mathrm{G} \times \mathfrak{g}^{*}}}{\longrightarrow} \mathcal{S}\left(\mathrm{G} \times \mathfrak{g}^{*}\right), & \operatorname{Op}_{\mathrm{G} \times \mathfrak{g}^{*}}\left(f_{1} \#{ }_{\mathrm{G} \times \mathfrak{g}^{*}} f_{2}\right):=\operatorname{Op}_{\mathrm{G} \times \mathfrak{g}^{*}}\left(f_{1}\right) \operatorname{Op}_{\mathrm{G} \times \mathfrak{g}^{*}}\left(f_{2}\right) .
\end{aligned}
$$

Denoting id $\otimes \mathscr{W}$ by $\mathfrak{W}$ one has

$$
\mathfrak{W}\left(f_{1} \#_{\mathbf{G} \times \mathfrak{g}^{*}} f_{2}\right)=\mathfrak{W}\left(f_{1}\right) \#_{\mathbf{G} \times \widehat{G}} \mathfrak{W J}\left(f_{2}\right), \quad \forall f_{1}, f_{2} \in \mathcal{S}\left(\mathbf{G} \times \mathfrak{g}^{*}\right) .
$$

One can write explicit (but rather complicated) formulae for these composition rules. In the case of $\mathrm{Op}_{\mathrm{G} \times \widehat{\mathrm{G}}}$, see [11] for a detailed discussion. Similarly for involutions. These ${ }^{*}$-algebras and their extensions will be studied separately. 
Remark 4.5. We make a formal statement about how convolution operators (in $\mathcal{S}(\mathrm{G}), L^{2}(\mathrm{G}$ ) or other function spaces on $G$ ) fit in the setting above; this can be made rigorous under suitable assumptions. Let us set

$$
\left[\operatorname{Conv}_{R}(w) u\right](x):=(u * w)(x)=\int_{\mathrm{G}} u(y) w\left(y^{-1} x\right) d \mathrm{~m}(y) .
$$

It is easy to show that, for suitable $B: \mathfrak{g}^{*} \rightarrow \mathbb{C}$, one has

$$
\operatorname{Conv}_{R}\left[\mathscr{F}_{\mathrm{G}, \mathfrak{g}^{*}}^{-1}(B)\right]=\operatorname{Conv}_{R}\left[\mathscr{F}_{\mathfrak{g}, \mathfrak{g}^{*}}^{-1}(B) \circ \log \right]=\mathrm{Op}_{\mathrm{G} \times \mathfrak{g}^{*}}(1 \otimes B) .
$$

Such left-invariant (or similar right-invariant) operators on various types of nilpotent Lie groups $\mathrm{G}$ were studied in detail in $[17,18,19,27,28,29]$ and other references.

On the other hand, in [26, Sect. 7.3] we proved that for convenient sections $b$ over $\widehat{G}$ one gets

$$
\operatorname{Conv}_{R}\left[\mathscr{F}_{\mathrm{G}, \widehat{\mathrm{G}}}^{-1}(b)\right]=\mathrm{Op}_{\mathrm{G} \times \widehat{\mathrm{G}}}(1 \otimes b) .
$$

Of course, this is compatible with Corollary 4.2. Writing (4.6) and (4.7) as

$$
\operatorname{Conv}_{R}(w)=\mathrm{Op}_{\mathrm{G} \times \mathfrak{g}^{*}}\left(1 \otimes\left[\mathscr{F}_{\mathrm{G}, \mathfrak{g}^{*}}(w)\right]\right)=\mathrm{Op}_{\mathrm{G} \times \widehat{\mathrm{G}}}\left(1 \otimes\left[\mathscr{F}_{\mathrm{G}, \widehat{\mathrm{G}}}(w)\right]\right),
$$

one could say that, in particular, $O \mathrm{p}_{\mathrm{G} \times \mathfrak{g}^{*}}$ and $O \mathrm{p}_{\mathrm{G} \times \widehat{\mathrm{G}}}$ are two different but related ways to study invariant operators through symbolic calculi. In the first case the symbols are scalar and defined on the dual of the Lie algebra, in the second case they are defined on the unitary dual of the group and are operator-valued. The same atitude towards non-invariant operators leads to the full quantizations (4.3) and (4.4) with "variable coefficients" pseudo-differential operators.

\subsection{The concrete Fourier transform and concrete quantizations}

The effect of the constructions and results described in Subsection 2.2 is that in the admissible case, for many purposes, one can replace the rather abstract and inaccessible measure space $(\widehat{G}, \widehat{m})$ by $\left(\mathfrak{z}_{\bullet}^{*}, \mu\right)$, where $\mathfrak{z}_{\bullet}^{*} \cong \omega_{\bullet}^{\dagger}$ is a subset of a finite-dimensional real vector space and $\mu$ a measure defined by an explicit density.

Taking advantage of Proposition 2.3, if $F$ is a function on $\widehat{\mathrm{G}}_{\bullet}$, we turn it into a function on $\mathfrak{z}_{\bullet}^{*}$ by $\tilde{\Xi}(F):=$ $F \circ \Xi$. Similarly, $\tilde{\Xi}^{-1}(G):=G \circ \Xi^{-1}$ is a function on $\widehat{G}$ if $G$ is a function on $\mathfrak{z}_{\bullet}^{*}$. The same works for sections in fiber bundles over the two spaces. Topological vector spaces of sections over $\widehat{G}_{\bullet}$ (as those defined over $\widehat{G}$ but insensible to removing the negligible subset $\widehat{G} \backslash \widehat{G}_{\bullet}$ ) are transferred to similar topological vector spaces of sections over $\mathfrak{z}_{\bullet}^{*}$. One has, for instance, the family of Banach spaces $\mathscr{B}^{p}\left(\mathfrak{z}_{\bullet}^{*}\right)$ indexed by $p \in[1, \infty)$, in particular, the Hilbert space

$$
\mathscr{B}^{2}\left(\mathfrak{z}_{\bullet}^{*}\right)=\int_{\mathfrak{z}_{\bullet}^{*}}^{\oplus} \mathbb{B}^{2}\left(\mathcal{H}_{\Xi(\mathcal{Z})}\right) 2^{d} d !|\operatorname{Pf}(\mathcal{Z})| d \mathcal{Z}
$$

Supposing that flat coadjoint orbits exist, with generic dimension $2 d$, one has

$$
\mathscr{F}_{\mathrm{G}_{\mathfrak{z}} *}:=\tilde{\Xi} \circ \mathscr{F}_{\mathrm{G}, \widehat{\mathrm{G}}}: L^{2}(\mathrm{G}) \rightarrow \mathscr{B}^{2}\left(\mathfrak{z}_{\bullet}^{*}\right)
$$

defined as

with inverse

$$
\left[\mathscr{F}_{\mathrm{G}, \mathfrak{z}^{*}}(u)\right](\mathcal{Z}):=\int_{\mathrm{G}} u(x) \xi_{\mathcal{Z}+\mathfrak{z}^{\dagger}}(x)^{*} d \mathrm{~m}(x),
$$

$$
\left(\mathscr{F}_{\mathrm{G}_{\mathfrak{3}}^{*}}^{-1} \Psi\right)(x):=2^{d} d ! \int_{\mathfrak{z}_{\bullet}^{*}} \operatorname{Tr}_{\xi_{\mathcal{Z}+\mathfrak{z}^{\dagger}}}\left[\Psi(\mathcal{Z}) \xi_{\mathcal{Z}+\mathfrak{z}^{\dagger}}(x)\right]|\operatorname{Pf}(\mathcal{Z})| d \mathcal{Z}
$$


Remark 4.6. Setting

$$
\mathscr{V}:=\mathscr{F}_{\mathrm{G}_{\mathfrak{z}}}{ }^{*} \circ \mathscr{F}_{\mathrm{G}, \mathfrak{g}^{*}}^{-1},
$$

one can write down explicit formulae, along the lines of Theorem 3.3. Basically, $\mathscr{V}$ consists in sending $B \in \mathscr{S}\left(\mathfrak{g}^{*}\right)$ into the family of restrictions $\left\{\left.B\right|_{\mathcal{Z}+\mathfrak{z}^{\dagger}} \mid \mathcal{Z} \in \omega_{\bullet}^{\dagger} \equiv \mathfrak{z}_{\bullet}^{*}\right\}$ and then into the family of operators

$$
\left\{\mathrm{b}(\mathcal{Z}):=\operatorname{Ped}_{\xi_{\mathcal{Z}+\mathfrak{z}^{\dagger}}}\left(\left.B\right|_{\mathcal{Z}+\mathfrak{z}^{\dagger}}\right) \mid \mathcal{Z} \in \omega_{\bullet}^{\dagger}\right\} \text {. }
$$

Its inverse is given by

$$
\left[\mathscr{V}^{-1}(\mathrm{~b})\right](\mathcal{X})=\int_{\omega} e^{i\langle Y \mid \mathcal{X}\rangle} \operatorname{Tr}_{\xi}\left[\mathrm{b}(\mathcal{Z}) \xi_{\mathcal{Z}}(\exp Y)^{*}\right] d \lambda_{\mathcal{Z}}(Y), \quad \forall \mathcal{X} \in \mathcal{Z}+\mathfrak{z}^{\dagger} \subset \mathfrak{g}^{*}
$$

A direct consequence is

Corollary 4.7. Choosing the concrete option, one can also define a quantization

$$
\begin{aligned}
& \mathrm{Op}_{\mathrm{G} \times \mathfrak{z}_{\bullet}^{*}}: \mathscr{B}^{2}\left(\mathrm{G} \times \mathfrak{z}_{\bullet}^{*}\right) \rightarrow \mathbb{B}^{2}\left[L^{2}(\mathrm{G})\right], \\
& {\left[\mathrm{Op}_{\mathrm{G} \times \mathfrak{z}_{\bullet}^{*}}(\Sigma) u\right](x)=2^{d} d ! \int_{\mathrm{G}} \int_{\mathfrak{z}_{\bullet}^{*}} \operatorname{Tr}\left[\Xi(\mathcal{Z})\left(y^{-1} x\right) \Sigma(x, \mathcal{Z})\right] u(y) d \mathrm{~m}(y)|\operatorname{Pf}(\mathcal{Z})| d \mathcal{Z} \text {. }}
\end{aligned}
$$

This one is connected to the quantizations (4.3) and (4.4) by

$$
\begin{gathered}
\mathrm{Op}_{\mathrm{G} \times \mathfrak{z}_{\bullet}^{*}}[(\mathrm{id} \otimes \mathscr{M})(f)]=\mathrm{Op}_{\mathrm{G} \times \widehat{\mathrm{G}}}[(\mathrm{id} \otimes \mathscr{W})(f)]=\mathrm{Op}_{\mathrm{G} \times \mathfrak{g}^{*}}(f), \quad f \in \mathcal{S}\left(\mathrm{G} \times \mathfrak{g}^{*}\right), \\
\mathrm{Op}_{\mathrm{G} \times \mathfrak{z}_{\bullet}^{*}}[(\mathrm{id} \otimes \tilde{\Xi})(\sigma)]=\mathrm{Op}_{\mathrm{G} \times \widehat{\mathrm{G}}}(\sigma), \quad \sigma \in \mathcal{S}(\mathrm{G} \times \widehat{\mathrm{G}}) .
\end{gathered}
$$

We recall that $[(\mathrm{id} \otimes \tilde{\Xi})(\sigma)](x, \mathcal{Z})=\sigma\left(x, \xi_{\mathcal{Z}+\mathfrak{z}^{\dagger}}\right)$ for every $x \in \mathrm{G}$ and $\mathcal{Z} \in \mathfrak{z}_{\bullet}^{*}$.

The next diagram tells us the story

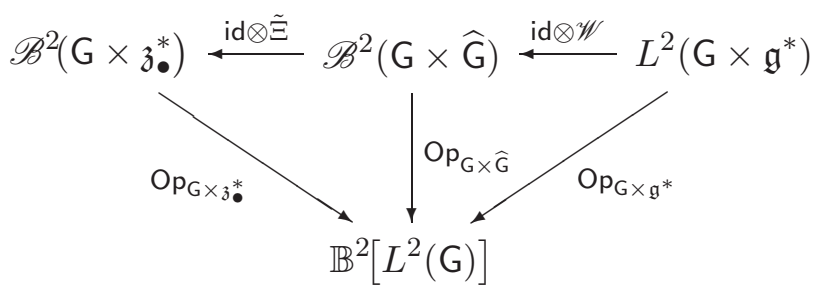

\section{Symbol classes for admissible graded groups}

In this section we discuss symbol classes of Hörmander's type in the case of graded nilpotent groups.

\subsection{Admissible graded Lie groups, their dilations and their Rockland operators}

We are going to review briefly some basic facts about graded Lie groups. Much more information can be found in [10, Ch. 4]; see also [14, 33].

Let $\mathrm{G}$ be a graded Lie group. Its Lie algebra can be written as a direct sum of vector subspaces

$$
\mathfrak{g}=\mathfrak{w}_{1} \oplus \cdots \oplus \mathfrak{w}_{l}
$$


where $\left[\mathfrak{w}_{i}, \mathfrak{w}_{k}\right] \subset \mathfrak{w}_{i+k}$ for every $i, k \in\{1, \ldots, l\}$, and where $l$ is such that $\mathfrak{w}_{i+k}=\{0\}$ for $i+k>l$. Then $\mathrm{G}$ is a connected and simply connected nilpotent Lie group. Let us set

$$
n_{k}:=\operatorname{dim} \mathfrak{w}_{k}, \quad n:=\operatorname{dim} \mathfrak{g}=n_{1}+n_{2}+\cdots+n_{l},
$$

and define the homogeneous dimension

$$
Q:=n_{1}+2 n_{2}+\cdots+\ln l
$$

We are going to use bases $\left\{X_{1}, \ldots, X_{n}\right\}$ of $\mathfrak{g}$ such that for every $k$ the $n_{k}$ vectors $\left\{X_{j} \mid n_{1}+\cdots+n_{k-1}<\right.$ $\left.j \leq n_{1}+\cdots+n_{k-1}+n_{k}\right\}$ generate $\mathfrak{w}_{k}$ (we set $n_{0}=0$ for convenience).

The multiplicative group $\left(\mathbb{R}_{+}, \cdot\right)$ acts by automorphisms (called dilations) of the Lie algebra (5.1) by

$$
\mathfrak{d i l}_{r}\left(Y_{(1)}, Y_{(2)}, \ldots, Y_{(l)}\right):=\left(r^{\nu_{1}} Y_{(1)}, r^{\nu_{2}} Y_{(2)} \ldots, r^{\nu_{l}} Y_{(l)}\right), \quad r \in \mathbb{R}_{+}, Y_{(k)} \in \mathfrak{w}_{k}, 1 \leq k \leq l .
$$

One has

$$
\mathfrak{d i l}_{r}\left(X_{j}\right)=r^{\nu_{j}} X_{j}, \quad r \in \mathbb{R}_{+}, 1 \leq j \leq n,
$$

in terms of the dilation weights $\nu_{j}$ if $n_{1}+\cdots+n_{k-1}<j \leq n_{1}+\cdots+n_{k}$. The dilations on the dual $\mathfrak{g}^{*}$ of the Lie algebra are defined by

$$
\left\langle X \mid \mathfrak{d i l} r_{r}^{*}(\mathcal{X})\right\rangle:=\left\langle\mathfrak{d i l}_{r^{-1}}(X) \mid \mathcal{X}\right\rangle, \quad X \in \mathfrak{g}, \mathcal{X} \in \mathfrak{g}^{*}, r \in \mathbb{R}_{+} .
$$

Since the graded groups are exponential, one can apply dilations on the group side, setting

$$
\operatorname{dil}_{r}(x):=\exp \left[\mathfrak{d i l}_{r}(\log x)\right], \quad x \in \mathrm{G}, r \in \mathbb{R}_{+} .
$$

This induces unitary operators on $\mathcal{H}:=L^{2}(\mathrm{G})$ by

$$
[\operatorname{Dil}(r) u](x):=r^{\frac{Q}{2}}\left(u \circ \operatorname{dil}_{r}\right)(x)=r^{\frac{Q}{2}} u\left(\operatorname{dil}_{r}(x)\right) .
$$

Finally, using the duality between $G$ and $\widehat{G}$ one makes $\left(\mathbb{R}_{+}, \cdot\right)$ act on the unitary dual by

$$
\left[\widehat{\operatorname{dil}}_{r}(\xi)\right](x):=\xi\left[\operatorname{dil}_{r^{-1}}(x)\right], \quad x \in \mathrm{G}, \xi \in \widehat{\mathrm{G}}, r>0 .
$$

The deliberate confusion between irreducible representations and their unitary equivalence classes is convenient and harmless.

A Rockland operator is a (say left) invariant differential operator $\mathcal{R}$ on $\mathrm{G}$ such that, for every non-trivial irreducible representation $\xi: G \rightarrow \mathbb{B}\left(\mathcal{H}_{\xi}\right)$, the operator $d \xi(\mathcal{R})$ is injective on the subspace $\mathcal{H}_{\xi}^{\infty}$ of all smooth vectors. We prefer them to be homogeneous and positive: the homogeneity reads, using (5.3)

$$
\operatorname{Dil}\left(r^{-1}\right) \mathcal{R} \operatorname{Dil}(r)=r^{\nu} \mathcal{R}, \quad \forall r \in \mathbb{R}_{+} .
$$

The degree of homogeneity $\nu$ is a multiple of any of the dilation weights. A left invariant homogeneous differential operator is hypoelliptic if and only if it is a Rockland operator [20, 10], see also [12] for a detailed discussion.

Convention: From now on we call simply Rockland operator a left invariant positive and homogeneous Rockland operator. These are the only ones appearing below; it is known that they exist on any graded group and, in fact, if such an operator exists on a connected simply connected Lie group, it has to be graded. 
Remark 5.1. Concrete examples of homogeneous degree $q=2 p$ are

$$
\mathcal{R}:=\sum_{j=1}^{n^{\prime}}(-1)^{\frac{p}{\nu_{j}}} Z_{j}^{2 \frac{p}{\nu_{j}}},
$$

where $\left\{Z_{j}\right\}_{j=1, \ldots, n}$ is a basis as in [10, Lemma 3.1.14] (see also [12]) and $p$ is a common multiple of the dilation weights. The basis is such that $Z_{j}$ is $\nu_{j}$-homogeneous, $Z_{1}, \ldots, Z_{n^{\prime}}$ generate $\mathfrak{g}$ as a Lie algebra, while $Z_{n^{\prime}+1}, \ldots, Z_{n}$ generate a vector space containing $[\mathfrak{g}, \mathfrak{g}]$.

Remark 5.2. Very concrete Rockland operators can be written down on stratified groups, which are graded groups for which $\mathfrak{w}_{1}$ in (5.1) generates $\mathfrak{g}$ as a Lie algebra. If $\left\{X_{1}, \ldots, X_{n_{1}}\right\}$ is a basis of the first stratum $\mathfrak{w}_{1}$, the left invariant 2-homogeneous negative operator

$$
\mathcal{L}:=X_{1}^{2}+\cdots+X_{n_{1}}^{2}
$$

is called a sub-Laplacian. Then $\mathcal{R}:=-\mathcal{L}$ is a Rockland operator.

It is important to note that Rockland operators are decomposable. For the theory of unbounded decomposable operators we refer to $[8,31]$. Let us fix a positive Rockland operator $\mathcal{R}$, homogeneous of order $\nu$. We also set $\mathcal{T}:=(\mathrm{id}+\mathcal{R})^{1 / \nu}$. The key fact is that both $\mathcal{R}$ and $\mathcal{T}$, acting in $L^{2}(\mathrm{G})$, become decomposable operators in $L^{2}(\widehat{\mathrm{G}}):=\int_{\widehat{\mathrm{G}}}^{\oplus} \mathcal{H}_{\xi} d \widehat{\mathrm{m}}(\xi)$ after applying unitary equivalence by an (extension of) the group Fourier transformation. Thus they are affiliated to the von Neumann algebra

$$
\mathcal{L}^{\infty}(\widehat{\mathrm{G}}):=\int_{\widehat{\mathrm{G}}}^{\oplus} \mathbb{B}\left(\mathcal{H}_{\xi}\right) d \widehat{\mathrm{m}}(\xi),
$$

which is isomorphically represented as the left group von Neumann algebra

$$
\mathbb{B}_{L}\left[L^{2}(\mathrm{G})\right]:=\left\{T \in \mathbb{B}\left[L^{2}(\mathrm{G})\right] \mid T \text { commutes with the left regular representation }\right\} .
$$

Setting

$$
\mathscr{R}:=\mathscr{F}_{\mathrm{G}, \widehat{\mathrm{G}}} \circ \mathcal{R} \circ \mathscr{F}_{\mathrm{G}, \widehat{\mathrm{G}}}^{-1}, \quad \mathscr{T}:=\mathscr{F}_{\mathrm{G}, \widehat{\mathrm{G}}} \circ \mathcal{T} \circ \mathscr{F}_{\mathrm{G}, \widehat{\mathrm{G}}}^{-1},
$$

one has for instance $(\mathscr{R} \phi)(\xi)=\mathscr{R}(\xi) \phi(\xi)$, where it is known that $\mathscr{R}(\xi)=d \xi(\mathcal{R})$ with domain $\mathcal{H}_{\xi}^{\nu}$, the represented Sobolev space of order $\nu$ defined at [10, 5.1.1]. Of course, each fiber operator acts continuously on the space of smooth vectors $d \xi(\mathcal{R}): \mathcal{H}_{\xi}^{\infty} \rightarrow \mathcal{H}_{\xi}^{\infty}$. For $\mathscr{T}$ one has

$$
\mathscr{T}(\xi)=\left(\operatorname{id}_{\xi}+\mathscr{R}(\xi)\right)^{1 / \nu}=\left(\operatorname{id}_{\xi}+d \xi(\mathcal{R})\right)^{1 / \nu}
$$

\subsection{The classes $S_{\rho, \delta}^{m}$}

Let us fix $q \in C_{\text {pol }}^{\infty}(\mathrm{G}):=\log \left[C_{\text {pol }}^{\infty}(\mathfrak{g})\right] \subset \mathcal{S}^{\prime}(\mathrm{G})$ (an intrinsic definition is also posible and other conditions or function spaces can be used). Then the operator of multiplication defined by $\operatorname{Mult}_{q}(u):=q u$ is welldefined linear and continuous in $\mathcal{S}(\mathrm{G})$ and in $\mathcal{S}^{\prime}(\mathrm{G})$. We also set

$$
\Delta_{q}:=\mathscr{F}_{\mathrm{G}, \widehat{\mathrm{G}}} \circ \mathrm{Mult}_{q} \circ \mathscr{F}_{\mathrm{G}, \widehat{\mathrm{G}}}^{-1} \in \mathbb{B}[\mathscr{S}(\widehat{\mathrm{G}})] \cap \mathbb{B}\left[\mathscr{S}^{\prime}(\widehat{\mathrm{G}})\right]
$$

and

$$
\Gamma_{q}:=\mathscr{F}_{\mathrm{G}, \mathfrak{g}^{*}} \circ \text { Mult }_{q} \circ \mathscr{F}_{\mathrm{G}, \mathfrak{g}^{*}}^{-1} \in \mathbb{B}\left[\mathcal{S}\left(\mathfrak{g}^{*}\right)\right] \cap \mathbb{B}\left[\mathcal{S}^{\prime}\left(\mathfrak{g}^{*}\right)\right]
$$


It follows directly from the definitions that

$$
\begin{aligned}
& \Gamma_{q}=\mathscr{F}_{\mathrm{G}, \mathfrak{g}^{*}} \circ \text { Mult }_{q} \circ \mathscr{F}_{\mathrm{G}, \mathfrak{g}^{*}}^{-1} \\
& =\left(\mathscr{F}_{\mathrm{G}, \mathfrak{g}^{*}} \circ \mathscr{F}_{\mathrm{G}, \widehat{\mathrm{G}}}^{-1}\right) \circ\left(\mathscr{F}_{\mathrm{G}, \widehat{\mathrm{G}}} \circ \operatorname{Mult}_{q} \circ \mathscr{F}_{\mathrm{G}, \widehat{\mathrm{G}}}^{-1}\right) \circ\left(\mathscr{F}_{\mathrm{G}, \widehat{\mathrm{G}}^{-}} \circ \mathscr{F}_{\mathrm{G}, \mathfrak{g}^{*}}^{-1}\right) \\
& =\mathscr{W}^{-1} \circ \Delta_{q} \circ \mathscr{W} \text {. }
\end{aligned}
$$

It can also be shown that $\Gamma_{q}$ is the operator of (commutative) convolution with $\mathscr{F}_{\mathrm{G}, \mathfrak{g}^{*}}(q)$ coming from the vector structure of the dual of the Lie algebra, but this will not be needed.

Certain special functions $q$ were used in $[10,12]$ to express the symbol class conditions. For multiindices $\alpha \in \mathbb{N}_{0}^{n}$, besides the usual length $|\alpha|:=\alpha_{1}+\alpha_{2}+\cdots+\alpha_{n}$, one also uses the homogeneous length $[\alpha]:=\sum_{k=1}^{n} \nu_{k} \alpha_{k}$, in terms of the dilation exponents $\nu_{1}, \ldots, \nu_{n}$. Also recall that a basis in $\mathrm{G}$ has been denoted by $\left\{X_{1}, \ldots, X_{n}\right\}$, leading to the differential operators $X_{x}^{\beta} \equiv X^{\beta}:=X_{1}^{\beta_{1}} \ldots X_{n}^{\beta_{n}}$. It is shown in $[10,12]$ that for every $\alpha \in \mathbb{N}_{0}^{n}$ there exists a unique homogeneous polynomial $q_{\alpha}: G \rightarrow \mathbb{R}$ of degree $[\alpha]$ such that

$$
\left(X^{\beta} q_{\alpha}\right)(\mathrm{e})=\delta_{\alpha, \beta}, \quad \forall \alpha, \beta \in \mathbb{N}_{0}^{n} .
$$

These polynomials are involved in Taylor developments and useful in writing down asymptotic developments for the $\mathrm{Op}_{\mathrm{G} \times \widehat{\mathrm{G}}}$ calculus. For $\alpha \in \mathbb{N}^{n}$, we set

$$
\tilde{q}_{\alpha}(x):=q_{\alpha}\left(x^{-1}\right), \Delta^{\alpha}:=\Delta_{\tilde{q}_{\alpha}} \text { and } \Gamma^{\alpha}:=\Gamma_{\tilde{q}_{\alpha}} .
$$

Assuming that the group $\mathrm{G}$ is both graded and admissible, let us fix a positive Rockland operator $\mathcal{R}$ homogeneous of degree $\nu$ and recall (5.4). By $\sup _{\xi \in \widehat{\mathrm{G}}}$ one denotes the essential supremum over $\widehat{\mathrm{G}}$ with respect to the Plancherel measure. For fixed numbers $m \in \mathbb{R}, \delta, \rho \in[0,1]$ such that $\rho \leq \delta$, the classes $S_{\rho, \delta}^{m}(\mathrm{G} \times \widehat{\mathrm{G}})$ were defined in $[10,12]$ by seminorm-conditions of the form

$$
\|\sigma\|_{S_{\rho, \delta ;(\alpha, \beta, \gamma)}^{m}}:=\sup _{x \in \mathbb{G}} \sup _{\xi \in \widehat{\mathrm{G}}}\left\|\mathscr{T}(\xi)^{-m+\rho[\alpha]-\delta[\beta]+\gamma}\left[\left(X_{x}^{\beta} \otimes \Delta^{\alpha}\right) \sigma\right](x, \xi) \mathscr{T}(\xi)^{-\gamma}\right\|_{\mathbb{B}\left(\mathcal{H}_{\xi}\right)}<\infty,
$$

involving all the multi-indices $\alpha, \beta, \gamma \in \mathbb{N}^{n}$.

We now write $\sigma=($ id $\otimes \mathscr{W}) f \in S_{\rho, \delta}^{m}(\mathrm{G} \times \widehat{\mathrm{G}})$ and try to see what the corresponding conditions on $f \in \mathscr{S}\left(\mathrm{G} \times \mathfrak{g}^{*}\right)$ are. We recall that, by Corollary 4.2, one has $((\mathrm{id} \otimes \mathscr{W}) g)(x, \xi)=\operatorname{Ped}_{\xi}\left(\left.g\right|_{\{x\} \times \Omega_{\xi}}\right)$. On the other hand, by (5.5),

$$
\left(X_{x}^{\beta} \otimes \Delta^{\alpha}\right) \circ(\mathrm{id} \otimes \mathscr{W})=(\mathrm{id} \otimes \mathscr{W}) \circ\left(X_{x}^{\beta} \otimes \Gamma^{\alpha}\right) .
$$

It follows immediately that

$$
\begin{aligned}
& \|f\|_{S_{\rho, \delta ;(\alpha, \beta, \gamma)}^{m}\left(\mathrm{G} \times \mathfrak{g}^{*}\right)}:=\|(\mathrm{id} \otimes \mathscr{W}) f\|_{S_{\rho, \delta ;(\alpha, \beta, \gamma)}^{m}} \\
& =\sup _{(x, \xi) \in \mathrm{G} \times \widehat{\mathrm{G}}}\left\|\mathscr{T}(\xi)^{-m+\rho[\alpha]-\delta[\beta]+\gamma} \operatorname{Ped}_{\xi}\left[\left.\left(\left(X_{x}^{\beta} \otimes \Gamma^{\alpha}\right) f\right)\right|_{\{x\} \times \Omega_{\xi}}\right] \mathscr{T}(\xi)^{-\gamma}\right\|_{\mathbb{B}\left(\mathcal{H}_{\xi}\right)} .
\end{aligned}
$$

Remark 5.3. The spaces of symbols

$$
S_{\rho, \delta}^{m}\left(\mathrm{G} \times \mathfrak{g}^{*}\right):=(\mathrm{id} \otimes \mathscr{W})^{-1} S_{\rho, \delta}^{m}(\mathrm{G} \times \widehat{\mathrm{G}}) \subset \mathscr{S}\left(\mathrm{G} \times \mathfrak{g}^{*}\right)
$$

can be defined along these lines, and they play the same role for the quantization $\mathrm{Op}_{\mathrm{G} \times \mathfrak{g}^{*}}$ as $S_{\rho, \delta}^{m}(\mathrm{G} \times \widehat{\mathrm{G}})$ played for the $\mathrm{Op}_{\mathrm{G} \times \widehat{\mathrm{G}}}$-calculus in $[10,12]$. This relationship allows one to transfer all results known for $O \mathrm{p}_{\mathrm{G} \times \widehat{G}}$ to this setting. Hopefully, in a future paper, we are going to undertake the non-trivial task of rephrasing the conditions (5.6) in a more tractable form, to write down explicit results for the pseudo-differential calculus on $\mathrm{G} \times \mathfrak{g}^{*}$, to compare it with existing (but only left or right invariant) calculi and to apply it to some concrete problems. 


\section{A four dimensional two-step stratified admissible group}

In this section we work out an example of a four dimensional two-step stratified admissible group and demonstrate the discussed quantizations in this setting.

\subsection{General facts}

Let $\mathfrak{g}:=\mathbb{R}^{4}$ be the Lie algebra with the bracket

$$
\left[(q, p, s, t),\left(q^{\prime}, p^{\prime}, s^{\prime}, t^{\prime}\right)\right]:=\left(0,0, q p^{\prime}-q^{\prime} p, \delta\left(q p^{\prime}-q^{\prime} p\right)\right),
$$

where $\delta \in \mathbb{R}$ is a real number. It is a four-dimensional step two Lie algebra with center

$$
\mathfrak{z}=\{0\} \times\{0\} \times \mathbb{R} \times \mathbb{R} .
$$

The canonical basis being denoted by $\{Q, P, S, T\}$, the single nontrivial bracket is

$$
[Q, P]=S+\delta T \text {. }
$$

Remark 6.1. (i) For convenience, we flipped the variables. A direct correspondence with the notations of previous sections, where the central variables stay first, would require the transformation $(q, p, s, t) \rightarrow$ $(s, t, q, p)$.

(ii) Here and subsequently, in vector spaces with specified basis one considers the Lebesgue measures canonically associated to these bases.

The corresponding connected simply connected Lie group is $G=\mathbb{R}^{4}$ with BCH-multiplication

$$
(q, p, s, t) \bullet\left(q^{\prime}, p^{\prime}, s^{\prime}, t^{\prime}\right)=\left(q+q^{\prime}, p+p^{\prime}, s+s^{\prime}+\frac{1}{2}\left(q p^{\prime}-q^{\prime} p\right), t+t^{\prime}+\frac{\delta}{2}\left(q p^{\prime}-q^{\prime} p\right)\right) .
$$

The unit is $\mathbf{0}:=(0,0,0,0)$ and the inverse of $(q, p, s, t)$ is $(-q,-p,-s,-t)$. In this realization, the maps exp and $\log$ are simply the identity of $\mathbb{R}^{4}$. Clearly $G$ is stratified with dilations

$$
(q, p, s, t) \rightarrow\left(r q, r p, r^{2} s, r^{2} t\right), \quad r>0 .
$$

Remark 6.2. One has two short exact sequences:

$$
1 \longrightarrow \mathbb{R}^{2} \equiv \mathrm{Z} \longrightarrow \mathrm{G} \stackrel{\Phi}{\longrightarrow} \mathbb{R}^{2} \longrightarrow 1
$$

with $\Phi(q, p, s, t):=(q, p)$, and 2-cocycle

$$
c_{1}: \mathbb{R}^{2} \times \mathbb{R}^{2} \rightarrow \mathrm{Z}, \quad c_{1}\left((q, p),\left(q^{\prime}, p^{\prime}\right)\right):=\left(\frac{1}{2}\left(q p^{\prime}-q^{\prime} p\right), \frac{\delta}{2}\left(q p^{\prime}-q^{\prime} p\right)\right),
$$

and

$$
1 \longrightarrow \mathbb{R} \longrightarrow \mathrm{G} \stackrel{\Psi}{\longrightarrow} \mathrm{H}_{1} \longrightarrow 1
$$

with $\Psi(q, p, s, t):=(q, p, s)$ and 2-cocycle

$$
c_{2}: \mathrm{H}_{1} \times \mathrm{H}_{1} \rightarrow \mathbb{R}, \quad c_{1}\left((q, p, s),\left(q^{\prime}, p^{\prime}, s^{\prime}\right)\right):=\frac{\delta}{2}\left(q p^{\prime}-q^{\prime} p\right) .
$$

The second one presents our nilpotent Lie group as a central extension of the 3-dimensional Heisenberg group by $\mathbb{R}$. It is split (actually a direct product) if and only if $\delta=0$. 


\subsection{The coadjoint action}

The adjoint action is

$$
\begin{aligned}
& \operatorname{Ad}_{\left(q_{0}, p_{0}, s_{0}, t_{0}\right)}(q, p, s, t)=\left(q_{0}, p_{0}, s_{0}, t_{0}\right) \bullet(q, p, s, t) \bullet\left(q_{0}, p_{0}, s_{0}, t_{0}\right)^{-1} \\
& \quad=\left(q_{0}+q, p_{0}+p, s_{0}+s+\frac{1}{2}\left(q_{0} p-q p_{0}\right), t_{0}+t+\frac{\delta}{2}\left(q_{0} p-q p_{0}\right)\right) \bullet\left(-q_{0},-p_{0},-s_{0},-t_{0}\right) \\
& \quad=\left(q, p, s+q_{0} p-q p_{0}, t+\delta\left(q_{0} p-q p_{0}\right)\right) .
\end{aligned}
$$

For the dual we set $\mathfrak{g}^{*}:=\mathbb{R}^{4} \ni(\rho, \vartheta, \varsigma, \tau) \equiv \rho \mathcal{Q}+\vartheta \mathcal{P}+\varsigma \mathcal{S}+\tau \mathcal{T}$ with duality

$$
\langle(q, p, s, t) \mid(\rho, \vartheta, \varsigma, \tau)\rangle:=q \rho+p \vartheta+s \varsigma+t \tau .
$$

The anihilator of the center is

$$
\mathfrak{z}^{\perp}=\mathbb{R} \times \mathbb{R} \times\{0\} \times\{0\} .
$$

The canonical bilinear form reads

$$
\begin{aligned}
\operatorname{Bil}_{(\rho, \vartheta, \varsigma, \tau)}\left((q, p, s, t),\left(q^{\prime}, p^{\prime}, s^{\prime}, t^{\prime}\right)\right) & =\left\langle\left[(q, p, s, t),\left(q^{\prime}, p^{\prime}, s^{\prime}, t^{\prime}\right)\right] \mid(\rho, \vartheta, \varsigma, \tau)\right\rangle \\
& =\left(q p^{\prime}-q^{\prime} p\right) \varsigma+\delta\left(q p^{\prime}-q^{\prime} p\right) \tau
\end{aligned}
$$

and it is non-degenerate when restricted to $\mathbb{R}^{2} \times\{(0,0)\}$.

Now we can compute the coadjoint action:

$$
\begin{aligned}
\left\langle(q, p, s, t) \mid \operatorname{Ad}_{\left(q_{0}, p_{0}, s_{0}, t_{0}\right)}^{*}(\rho, \vartheta, \varsigma, \tau)\right\rangle=\left\langle\operatorname{Ad}_{\left(-q_{0},-p_{0},-s_{0},-t_{0}\right)}(q, p, s, t) \mid(\rho, \vartheta, \varsigma, \tau)\right\rangle \\
=\left\langle\left(q, p, s-q_{0} p+q p_{0}, t-\delta\left(q_{0} p-q p_{0}\right)\right) \mid(\rho, \vartheta, \varsigma, \tau)\right\rangle \\
=q\left(\rho+p_{0} \varsigma+\delta p_{0} \tau\right)+p\left(\vartheta-q_{0} \varsigma-\delta q_{0} \tau\right)+s \varsigma+t \tau
\end{aligned}
$$

meaning that

$$
\begin{aligned}
\operatorname{Ad}_{\left(q_{0}, p_{0}, s_{0}, t_{0}\right)}^{*}(\rho, \vartheta, \varsigma, \tau) & =\left(\rho+p_{0} \varsigma+\delta p_{0} \tau, \vartheta-q_{0} \varsigma-\delta q_{0} \tau, \varsigma, \tau\right) \\
& =(\rho, \vartheta, \varsigma, \tau)+\left(p_{0}[\varsigma+\delta \tau],-q_{0}[\varsigma+\delta \tau], 0,0\right) .
\end{aligned}
$$

The fixed points have all the form

$$
(\rho, \vartheta,-\delta \tau, \tau), \quad \rho, \vartheta, \tau \in \mathbb{R} .
$$

If $\varsigma \neq-\delta \tau$, the coadjoint orbit passing through $(\rho, \vartheta, \varsigma, \tau)$ is flat and 2 - dimensional:

$$
\Omega_{(\rho, \vartheta, \varsigma, \tau)}=(\rho, \vartheta, \varsigma, \tau)+\mathbb{R}^{2} \times\{(0,0)\}=(\rho, \vartheta, \varsigma, \tau)+\mathfrak{z}^{\perp}=(0,0, \varsigma, \tau)+\mathfrak{z}^{\perp} .
$$

It only depends on $(\varsigma, \tau)$ and can be written in the form

$$
\Omega_{(\varsigma, \tau)}=(0,0, \varsigma, \tau)+\mathbb{R}^{2} \times\{(0,0)\}=\{(\rho, \vartheta, \varsigma, \tau) \mid \rho, \vartheta \in \mathbb{R}\} .
$$

The restriction of $\operatorname{Ad}_{\left(q_{0}, p_{0}, s_{0}, t_{0}\right)}^{*}$ to such an orbit is the translation by $\left(p_{0} \varsigma+\delta p_{0} \tau,-q_{0} \varsigma-\delta q_{0} \tau, 0,0\right)$, so the invariant measures are all proportional to the transported 2-dimensional Lebesgue measure $d \rho d \vartheta$ (cf. [6, 
Th. 1.2.12] for a more general statement). So they can be written as $c(\varsigma, \tau) d \rho d \vartheta$ for positive numbers $c(\varsigma, \tau)$. The good normalisation, leading to the canonical measure $d \gamma_{\Omega_{(\varsigma, \tau)}} \equiv d \gamma_{(\varsigma, \tau)}$ used repeatedly above, is

$$
d \gamma_{(\varsigma, \tau)}(\rho, \vartheta)=(2|\varsigma+\delta \tau|)^{-1} d \rho d \vartheta .
$$

This can be seen rather easily by inspecting [6, 4.3], but it is also explained in Remark 6.3.

The isotropy group and algebra of the generic points $(\rho, \vartheta, \varsigma, \tau)$ are, respectively,

$$
\mathrm{G}_{(\rho, \vartheta, \varsigma, \tau)}=\{(0,0)\} \times \mathbb{R}^{2}, \quad \mathfrak{g}_{(\rho, \vartheta, \varsigma, \tau)}=\{(0,0)\} \times \mathbb{R}^{2}=\mathfrak{z} .
$$

According to the general theory or to (6.1), the canonical bilinear form can be seen as a map from $\mathfrak{z}^{*} \cong\{(0,0)\} \times \mathbb{R}^{2} \equiv \mathbb{R}^{2}$ to the space of antisymmetric bilinear (non-degenerate) forms on the common pre-dual $\omega=\mathbb{R}^{2} \times\{(0,0)\} \equiv \mathbb{R}^{2}$, given by

$$
\operatorname{Bil}_{(\varsigma, \tau)}\left((q, p),\left(q^{\prime}, p^{\prime}\right)\right)=\left(q p^{\prime}-q^{\prime} p\right) \varsigma+\delta\left(q p^{\prime}-q^{\prime} p\right) \tau
$$

The determinant is $\operatorname{det} \operatorname{Bil}_{(\varsigma, \tau)}=(\varsigma+\delta \tau)^{2}$, so

$$
\operatorname{Pf}(\sigma, \tau)=\varsigma+\delta \tau=0 \quad \Longleftrightarrow \quad \varsigma=-\delta \tau,
$$

and the irreducible representations that are square integrable modulo the center (or, equivalently, the flat orbits) are labelled by

$$
\mathfrak{z}_{\bullet}^{*}=\left\{(\varsigma, \tau) \in \mathbb{R}^{2} \mid \varsigma \neq-\delta \tau\right\} .
$$

The transported Plancherel measure on $\mathfrak{z}^{*}$ is concentrated on this set and it has a density with respect to the 2-dimensional Lebesgue measure:

$$
d \mu(\varsigma, \tau)=2|\operatorname{Pf}(\varsigma, \tau)| d \sigma d \tau=2|\varsigma+\delta \tau| d \varsigma d \tau .
$$

Remark 6.3. One can combine this form of the Plancherel measure with Lemma 3.5 to compute the canonical measures on our flat coadjoint orbits. Using the concrete realisation, (3.6) becomes

$$
\int_{\mathbb{R}^{2}}\left[\int_{\Omega_{(\varsigma, \tau)}} C(\rho, \vartheta, \varsigma, \tau) d \gamma_{(\varsigma, \tau)}(\rho, \vartheta)\right] 2|\varsigma+\delta \tau| d \sigma d \tau=\int_{\mathfrak{g}^{*}} C(\rho, \vartheta, \varsigma, \tau) d \rho d \vartheta d \varsigma d \tau
$$

from which (6.2) follows.

\subsection{Irreducible representations}

The way to construct the irreducible representations of $\mathrm{G}$ is exposed in a general setting in [6, Sect. 2] and will be applied without many comments.

We first determine the irreducible representations attached to the fixed points. If $(\rho, \vartheta,-\delta \tau, \tau)$ is such a fixed point, the entire Lie algebra $\mathfrak{g}$ is polarizing (maximal subordinate). The associated character

$$
\chi_{(\rho, \vartheta,-\delta \tau, \tau)}: \mathbf{G} \equiv \mathfrak{g} \rightarrow \mathbb{C}, \quad \chi_{(\rho, \vartheta,-\delta \tau, \tau)}(q, p, s, t):=e^{i\langle(q, p, s, t) \mid(\rho, \vartheta,-\delta \tau, \tau)\rangle}=e^{i(q \rho+p \vartheta+(t-\delta s) \tau)}
$$

is the representation we were looking for. We recall that these representations have no contribution to the Plancherel measure. If $u \in L^{1}(\mathrm{G})$, then its group Fourier tranform computed in these characters (just 
complex numbers) can be expressed as a restriction of the 4-dimensional Euclidean Fourier transform:

$$
\begin{aligned}
\left(\mathscr{F}_{\mathrm{G}, \widehat{\mathrm{G}}} u\right)\left(\chi_{(\rho, \vartheta,-\delta \tau, \tau)}\right) & =\int_{\mathbb{R}^{4}} u(q, p, s, t) \chi_{(\rho, \vartheta,-\delta \tau, \tau)}(-q,-p,-s,-t) d q d p d s d t \\
& =\int_{\mathbb{R}^{4}} u(q, p, s, t) e^{-i(q \rho+p \vartheta+(t-\delta s) \tau)} d q d p d s d t \\
& =\left(\mathcal{F}_{\mathbb{R}^{4}} u\right)(\rho, \vartheta,-\delta \tau, \tau) .
\end{aligned}
$$

In search of the irreducible representations corresponding to the flat orbits, we fix the (Abelian) Lie subalgebra

$$
\mathfrak{m}:=\{0\} \times \mathbb{R}^{3}=\operatorname{Span}(P, S, T) .
$$

It is clearly polarizing for all these flat orbits, since it has the right dimension $\operatorname{dim} \mathfrak{m}=\frac{1}{2}(\operatorname{dim} \mathfrak{g}+\operatorname{dim} \mathfrak{z})$ and, by (6.1), one has

$$
\operatorname{Bil}_{(\rho, \vartheta, \varsigma, \tau)}\left((0, p, s, t),\left(0, p^{\prime}, s^{\prime}, t^{\prime}\right)\right)=0 .
$$

If $(\varsigma, \tau) \in \mathfrak{z}_{\bullet}^{*}$, i.e. $\varsigma \neq-\delta \tau$, the character

$$
\chi_{(\varsigma, \tau)}: \mathrm{M} \equiv \mathfrak{m} \rightarrow \mathbb{T}, \quad \chi_{(\varsigma, \tau)}(0, p, s, t)=e^{i\langle(0, p, s, t) \mid(0,0, \varsigma, \tau)\rangle}=e^{i(s \varsigma+t \tau)}
$$

serves to induce the irreducible representation

$$
\pi_{(\varsigma, \tau)}:=\operatorname{lnd}\left(\mathrm{M} \uparrow \mathrm{G} ; \chi_{(\varsigma, \tau)}\right): \mathrm{G} \rightarrow \mathbb{B}\left(\mathcal{H}_{(\varsigma, \tau)}\right) .
$$

As model Hilbert space $\mathcal{H}_{(\sigma, \tau)}$ we are going to use $L^{2}(\mathrm{G} / \mathrm{M}) \equiv L^{2}(\mathbb{R})$. We will need the computation

$$
\begin{aligned}
\left(q_{0}, 0,0,0\right) \bullet(q, p, s, t) & =\left(q_{0}+q, p, s+\frac{1}{2} q_{0} p, t+\frac{\delta}{2} p_{0} q\right) \\
& =\left(0, p, s+q_{0} p+\frac{1}{2} q p, t+\delta q_{0} p+\frac{\delta}{2} q p\right) \bullet\left(q_{0}+q, 0,0,0\right) .
\end{aligned}
$$

Then the general theory gives for $\varphi \in L^{2}(\mathbb{R})$ the expression of the corresponding irreducible representation

$$
\begin{aligned}
{\left[\pi_{(\varsigma, \tau)}(q, p, s, t) \varphi\right]\left(q_{0}\right) } & =\chi_{(\varsigma, \tau)}\left(0, p, s+q_{0} p+\frac{1}{2} q p, t+\delta q_{0} p+\frac{\delta}{2} q p\right) \varphi\left(q_{0}+q\right) \\
& =e^{i\left[s \varsigma+t \tau+\left(q_{0} p+\frac{1}{2} q p\right)(\varsigma+\delta \tau)\right]} \varphi\left(q_{0}+q\right) .
\end{aligned}
$$

Remark 6.4. Let $r>0$; denoting by dil $_{\sqrt{r}}$ the unitary dilation operator $\left(\operatorname{dil}_{\sqrt{r}} \varphi\right)\left(q_{0}\right):=\sqrt{r} \varphi\left(\sqrt{r} q_{0}\right)$ in $L^{2}(\mathbb{R})$, one checks easily that

$$
\pi_{(r \varsigma, r \tau)}(q, p, s, t) \circ \operatorname{dil}_{\sqrt{r}}=\operatorname{dil}_{\sqrt{r}} \circ \pi_{(\varsigma, \tau)}(\sqrt{r} q, \sqrt{r} p, r s, r t)=\operatorname{dil}_{\sqrt{r}} \circ \pi_{(\varsigma, \tau)}[\sqrt{r} \cdot(q, p, s, t)] .
$$

Setting $\mathfrak{q}$ for the operator of multiplication with the variable in $L^{2}(\mathbb{R})$, i.e. $(\mathfrak{q} \varphi)(q):=q \varphi(q)$, the four infinitesimal generators are

$$
d \pi_{(\varsigma, \tau)}(Q)=\partial, \quad d \pi_{(\varsigma, \tau)}(P)=i(\varsigma+\delta \tau) \mathfrak{q}, \quad d \pi_{(\varsigma, \tau)}(S)=i \sigma \mathbf{l d}, \quad d \pi_{(\varsigma, \tau)}(T)=i \tau \mathbf{l d} .
$$

Thus the repesented version of the canonical sub-Laplacian $\mathcal{L}:=Q^{2}+P^{2}$ is

$$
d \pi_{(\varsigma, \tau)}(\mathcal{L})=\partial^{2}-(\varsigma+\delta \tau)^{2} \mathfrak{q}^{2} .
$$

The general theory tells us that $\mathcal{H}_{(\varsigma, \tau)}^{\infty}=\mathcal{S}(\mathbb{R})$. 


\subsection{The group Fourier transform and the Pedersen calculus}

We are going to make use of Weyl's quantization [13] with a parameter $\lambda \in \mathbb{R}_{\bullet}:=\mathbb{R} \backslash\{0\}$ in one dimension

$$
\left[\operatorname{Weyl}_{\lambda}(\gamma) \varphi\right]\left(q_{0}\right):=\int_{\mathbb{R}} \int_{\mathbb{R}} e^{i\left(q_{0}-q\right) \eta} \gamma\left(\eta, \lambda \frac{q_{0}+q}{2}\right) \varphi(q) d q d \eta
$$

In computing the group Fourier transform and the Pedersen quantization, we only treat the (generic) flat orbits $\Omega_{(\varsigma, \tau)}=(0,0, \varsigma, \tau)+\mathbb{R}^{2} \times\{(0,0)\}$, where $\varsigma \neq-\delta \tau$. One denotes by $\mathcal{F}_{\mathbb{R}^{k}}$ the usual Euclidean Fourier transform in $\mathbb{R}^{k}$.

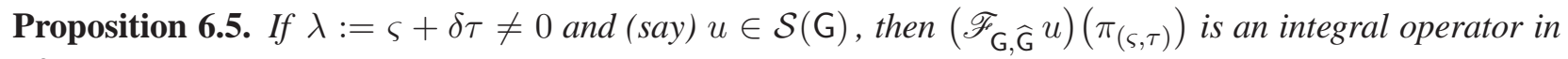
$L^{2}(\mathbb{R})$ with kernel

$$
\kappa_{(\varsigma, \tau)}^{u}\left(q_{0}, q\right):=\left[\left(\mathbf{I d} \otimes \mathcal{F}_{\mathbb{R}^{3}}\right) u\right]\left(q_{0}-q, \frac{\lambda}{2}\left(q_{0}+q\right), \varsigma, \tau\right)
$$

and also a Weyl $\lambda$-pseudo-differential operator with symbol $\left.\left(\mathcal{F}_{\mathbb{R}^{4}} u\right)\right|_{\mathbb{R}^{2} \times\{(\varsigma, \tau)\}}$, i.e. it is given by

$$
\left[\left(\mathscr{F}_{\mathrm{G}, \widehat{\mathrm{G}}} u\right)\left(\pi_{(\varsigma, \tau)}\right) \varphi\right]\left(q_{0}\right)=\int_{\mathbb{R}} \int_{\mathbb{R}} e^{i\left(q_{0}-q\right) \eta}\left(\mathcal{F}_{\mathbb{R}^{4}} u\right)\left(\eta, \frac{\varsigma+\delta \tau}{2}\left(q_{0}+q\right), \varsigma, \tau\right) \varphi(q) d q .
$$

Proof. If $u \in L^{1}(\mathrm{G})$ and $\varphi \in L^{2}(\mathbb{R})$, then

$$
\begin{aligned}
{\left[\left(\mathscr{F}_{\mathrm{G}, \widehat{\mathrm{G}}} u\right)\right.} & \left.\left(\pi_{(\varsigma, \tau)}\right) \varphi\right]\left(q_{0}\right)=\int_{\mathbb{R}^{4}} u(q, p, s, t)\left[\pi_{(\varsigma, \tau)}(-q,-p,-s,-t) \varphi\right]\left(q_{0}\right) d q d p d s d t \\
& =\int_{\mathbb{R}^{4}} u(q, p, s, t) e^{-i\left[s \varsigma+t \tau+\left(q_{0} p-\frac{1}{2} q p\right)(\varsigma+\delta \tau)\right]} \varphi\left(q_{0}-q\right) d q d p d s d t \\
& =\int_{\mathbb{R}}\left[\left(\mathrm{Id} \otimes \mathcal{F}_{\mathbb{R}^{3}}\right) u\right]\left(q_{0}-q, \frac{\varsigma+\delta \tau}{2}\left(q_{0}+q\right), \sigma, \tau\right) \varphi(q) d q \\
& =\int_{\mathbb{R}} \int_{\mathbb{R}} e^{i\left(q_{0}-q\right) \eta}\left(\mathcal{F}_{\mathbb{R}^{4}} u\right)\left(\eta, \frac{\varsigma+\delta \tau}{2}\left(q_{0}+q\right), \varsigma, \tau\right) \varphi(q) d q,
\end{aligned}
$$

finishing the proof.

Proposition 6.6. If $(\varsigma, \tau) \in \mathfrak{z}_{\bullet}^{*}$, then $\operatorname{Ped}_{\Omega_{(\varsigma, \tau)}} \equiv \operatorname{Ped}_{(\varsigma, \tau)}$ only depends on the combination $\lambda:=\varsigma+\delta \tau \neq 0$ and one has

$$
\operatorname{Ped}_{(\varsigma, \tau)}=(2|\varsigma+\delta \tau|)^{-1} \text { Weyl }_{\varsigma+\delta \tau} .
$$

Proof. We start with the Fourier transform adapted to the coadjoint orbit. For any $\Psi \in \mathcal{S}\left(\Omega_{(\varsigma, \tau)}\right)$, seen as a function of $(\rho, \vartheta) \in \mathbb{R}^{2}$, and for any $(q, p, s, t) \in \mathfrak{g}$, we have by (6.2) that

$$
\hat{\Psi}(q, p, s, t):=\int_{\Omega_{(\varsigma, \tau)}} e^{-i\langle(q, p, s, t) \mid(\rho, \vartheta, \varsigma, \tau)\rangle} \Psi(\rho, \vartheta)(2|\varsigma+\delta \tau|)^{-1} d \rho d \vartheta
$$

and then, since the common predual is $\omega=\mathbb{R}^{2} \times\{(0,0)\}$,

$$
\left[\mathrm{F}_{\Omega_{(\varsigma, \tau)}}(\Psi)\right](q, p)=\hat{\Psi}(q, p, 0,0)=(2|\varsigma+\delta \tau|)^{-1} \int_{\mathbb{R}^{2}} e^{-i(q \rho+p \vartheta)} \Psi(\rho, \vartheta) d \rho d \vartheta
$$


so (after some identifications), we essentially arrived once more at the Euclidean Fourier transform. Then, taking into account (6.4) and the definition of the Pedersen quantization, for $\Psi \in \mathcal{S}\left(\Omega_{(\varsigma, \tau)}\right)$ one can write

$$
\begin{aligned}
\operatorname{Ped}_{(\varsigma, \tau)}(\Psi) & \varphi]\left(q_{0}\right)=(2|\varsigma+\delta \tau|)^{-1} \int_{\mathbb{R}^{2}} \int_{\mathbb{R}^{2}} e^{-i(q \rho+p \vartheta)} \Psi(\rho, \vartheta)\left[\pi_{(\varsigma, \tau)}(q, p, 0,0) \varphi\right]\left(q_{0}\right) d q d p d \rho d \vartheta \\
& =(2|\varsigma+\delta \tau|)^{-1} \int_{\mathbb{R}^{2}} \int_{\mathbb{R}^{2}} e^{-i(q \rho+p \vartheta)} \Psi(\rho, \vartheta) e^{i\left(q_{0} p+\frac{1}{2} q p\right)(\varsigma+\delta \tau)} \varphi\left(q_{0}+q\right) d p d q d \rho d \vartheta \\
& =|2 \lambda|^{-1} \int_{\mathbb{R}^{3}}\left(\int_{\mathbb{R}} e^{i p\left[\lambda\left(q_{0}+\frac{1}{2} q\right)-\vartheta\right]} d p\right) e^{-i q \rho} \Psi(\rho, \vartheta) \varphi\left(q_{0}+q\right) d q d \rho d \vartheta \\
& =|2 \lambda|^{-1} \int_{\mathbb{R}} \int_{\mathbb{R}} e^{-i q \rho} \Psi\left(\rho, \lambda\left(q_{0}+q / 2\right)\right) \varphi\left(q_{0}+q\right) d p d \rho \\
& =|2 \lambda|^{-1} \int_{\mathbb{R}} \int_{\mathbb{R}} e^{i\left(q_{0}-q\right) \rho} \Psi\left(\rho, \lambda \frac{q_{0}+q}{2}\right) \varphi(q) d q d \rho .
\end{aligned}
$$

The forth equality is a formal but easy to justify standard fact.

\subsection{Quantization}

Therefore, as in Corollary 4.7, the concrete form of the global group quantization is

$$
\begin{gathered}
{\left[\operatorname{Op}_{\left.\mathrm{G}_{\mathbf{z}_{\mathbf{3}}^{*}}(\Sigma) u\right]\left(q^{\prime}, p^{\prime}, s^{\prime}, t^{\prime}\right)}\right.} \\
=2 \int_{\mathbb{R}^{4}} \int_{\mathbb{R}^{2}} \operatorname{Tr}\left[\pi_{(\varsigma, \tau)}\left(q^{\prime}-q, p^{\prime}-p, s^{\prime}-s-\frac{1}{2}\left(q p^{\prime}-q^{\prime} p\right), t^{\prime}-t-\frac{\delta}{2}\left(q p^{\prime}-q^{\prime} p\right)\right) \Sigma\left(q^{\prime}, p^{\prime}, s^{\prime}, t^{\prime} ; \varsigma, \tau\right)\right] \\
u(q, p, s, t)|\varsigma+\delta \tau| d \varsigma d \tau d q d p d s d t .
\end{gathered}
$$

On the other hand, the quantization on $G \times \mathfrak{g}^{*}$ indicated in (4.3) reads now

$$
\begin{gathered}
{\left[\mathrm{Op}_{\mathrm{G} \times \mathfrak{g}^{*}}(f) u\right]\left(q^{\prime}, p^{\prime}, s^{\prime}, t^{\prime}\right)=\int_{\mathbb{R}^{4}} \int_{\mathbb{R}^{4}} e^{i\left[\left(q^{\prime}-q\right) \rho+\left(p^{\prime}-p\right) \vartheta+\left(s^{\prime}-s\right) \varsigma+\left(t^{\prime}-t\right) \tau\right]} e^{-\frac{i}{2}\left(q p^{\prime}-q^{\prime} p\right)(\sigma+\delta \tau)}} \\
f\left(q^{\prime}, p^{\prime}, s^{\prime}, t^{\prime} ; \rho, \vartheta, \varsigma, \tau\right) u(q, p, s, t) d q d p d s d t d \rho d \vartheta d \varsigma d \tau .
\end{gathered}
$$

Remark 6.7. By (3.8) and (6.2), one has

$$
\begin{aligned}
& \operatorname{Tr}\left[\pi_{(\sigma, \tau)}\left(q^{\prime}-q, p^{\prime}-p, s^{\prime}-s-\frac{1}{2}\left(q p^{\prime}-q^{\prime} p\right), t^{\prime}-t-\frac{\delta}{2}\left(q p^{\prime}-q^{\prime} p\right)\right) \operatorname{Ped}{ }_{(\varsigma, \tau)}\left(f\left(q^{\prime}, p^{\prime}, s^{\prime}, t^{\prime} ; \cdot, \cdot, \sigma, \tau\right)\right)\right] \\
& =(2|\sigma+\delta \tau|)^{-1} \int_{\mathbb{R}^{2}} e^{i\left\langle q^{\prime}-q, p^{\prime}-p, s^{\prime}-s-\frac{1}{2}\left(q p^{\prime}-q^{\prime} p\right), t^{\prime}-t-\frac{\delta}{2}\left(q p^{\prime}-q^{\prime} p\right) \mid(\rho, \vartheta, \varsigma, \tau)\right\rangle} f\left(q^{\prime}, p^{\prime}, s^{\prime}, t^{\prime} ; \rho, \vartheta, \sigma, \tau\right) d \rho d \vartheta \\
& =(2|\sigma+\delta \tau|)^{-1} \int_{\mathbb{R}^{2}} e^{i\left[\left(q^{\prime}-q\right) \rho+\left(p^{\prime}-p\right) \vartheta+\left(s^{\prime}-s\right) \sigma+\left(t^{\prime}-t\right) \tau\right]} e^{-\frac{i}{2}\left(q p^{\prime}-q^{\prime} p\right)(\varsigma+\delta \tau)} f\left(q^{\prime}, p^{\prime}, s^{\prime}, t^{\prime} ; \rho, \vartheta, \sigma, \tau\right) d \rho d \vartheta .
\end{aligned}
$$

Replacing this in (6.5), for

$$
\Sigma\left(q^{\prime}, p^{\prime}, s^{\prime}, t^{\prime} ; \sigma, \tau\right)=\operatorname{Ped}_{(\sigma, \tau)}\left(f\left(q^{\prime}, p^{\prime}, s^{\prime}, t^{\prime} ; \cdot, \cdot, \sigma, \tau\right)\right),
$$

one recovers (6.6). This is a confirmation of Corollary 4.2 in this simple particular case.

\section{Appendix: Admissible graded Lie algebras with one-dimensional center}

Here we discuss several examples of admissible graded Lie groups and the appearing elements of their representatons. 


\subsection{Automorphisms and Lie algebras with one-dimensional center}

An automorphism of the Lie algebra generates many other automorphisms that interact well with the coadjoint picture. Let $\mathfrak{c}: \mathfrak{g} \rightarrow \mathfrak{g}$ be such an automorphism; then another one is defined by

$$
\mathfrak{c}^{*}: \mathfrak{g}^{*} \rightarrow \mathfrak{g}^{*}, \quad\left\langle X \mid \mathfrak{c}^{*}(\mathcal{X})\right\rangle:=\left\langle\mathfrak{c}^{-1}(X) \mid \mathcal{X}\right\rangle .
$$

The exponential map being an isomorphism, one can apply the automorphism on the group side, setting

$$
\mathrm{c}(x):=\exp [\mathfrak{c}(\log x)], \quad x \in \mathrm{G} .
$$

Finally, using the duality between $\mathrm{G}$ and $\widehat{\mathrm{G}}$ one makes the automorphism act on the unitary dual by

$$
\widehat{\mathrm{c}}(\xi)](x):=\xi[\mathrm{c}(x)], \quad x \in \mathrm{G}, \xi \in \widehat{\mathrm{G}} .
$$

The deliberate confusion between irreducible representations and their unitary equivalence classes is harmless. If we adopt the representation point of view, note that the Hilbert spaces of $\xi$ and $\widehat{c}(\xi)$ are the same.

Lemma 7.1. Let $\mathfrak{c}$ be an automorphisms of the Lie algebra $\mathfrak{g}$.

(i) For every $x \in \mathrm{G}$ one has

$$
\operatorname{Ad}_{x} \circ \mathfrak{c}=\mathfrak{c} \circ \operatorname{Ad}_{c^{-1}(x)}, \quad \mathfrak{c}^{*} \circ \operatorname{Ad}_{x}^{*}=\operatorname{Ad}_{c^{-1}(x)}^{*} \circ \mathfrak{c}^{*}
$$

(ii) The automorhism $\mathfrak{c}^{*}$ sends coadjoint orbits in coadjoint orbits.

(iiii) If $\mathfrak{m}$ is a polarizing subalgebra for $\mathcal{U} \in \mathfrak{g}$, then $\mathfrak{c}(\mathfrak{m})$ is a polarizing subalgebra for $\mathfrak{c}^{*}(\mathcal{U})$.

(iv) If $\mathrm{G}$ is admissible, the automorhism $\mathfrak{c}^{*}$ sends flat coadjoint orbits in flat coadjoint orbits.

Proof. (i) Using (7.1) and notations from Subsection 2.1, one has

$$
\begin{aligned}
\operatorname{Ad}_{x} \circ \mathfrak{c} & =\log \circ \operatorname{inn}_{x} \circ \exp \circ \mathfrak{c}=\log \circ \operatorname{inn}_{x} \circ \mathrm{c} \circ \exp \\
& =\log \circ \mathrm{c} \circ \operatorname{inn}_{c^{-1}(x)} \circ \exp =\mathfrak{c} \circ \log \circ \operatorname{inn}_{c^{-1}(x)} \circ \exp \\
& =\mathfrak{c} \circ \operatorname{Ad}_{c^{-1}(x)},
\end{aligned}
$$

which shows the first identity in (7.2). The second one follows by duality.

(ii) The second identity in (7.2) implies immediately that

$$
\mathfrak{c}^{*}\left(\Omega_{\mathcal{U}}\right)=\Omega_{\mathfrak{c}^{*}(\mathcal{U})}, \quad \forall \mathcal{U} \in \mathfrak{g}^{*}
$$

(iii) Straightforward proof: see [6, Prop. 1.3.6].

(iv) Recall that the flat orbits are of the form $\Omega=\mathcal{Z}+\mathfrak{z}^{\perp}$, with $\mathcal{Z} \in \mathfrak{z}_{\bullet}^{*}$. So, by (7.3), it is enough to show that $\mathfrak{c}^{*}: \mathfrak{g}^{*} \rightarrow \mathfrak{g}^{*}$ leaves $\mathfrak{z}_{\bullet}^{*}$ invariant. Clearly $\mathfrak{z}^{*}$ is invariant under the automorphism $\mathfrak{c}^{*}$ : use for instance the fact that the center $\mathfrak{z}$ is invariant under any automorphism of $\mathfrak{g}$. The points $\mathcal{Z} \in \mathfrak{z}_{\bullet}^{*}$ are characterized by the condition $\operatorname{Pf}(\mathcal{Z})^{2}=\operatorname{det}\left(\operatorname{Bil}_{\mathcal{Z}}\right) \neq 0$. But

$$
\operatorname{Bil}_{\mathfrak{c}^{*}(\mathcal{Z})}=\operatorname{Bil}_{\mathcal{Z}} \circ\left(\mathfrak{c}^{-1} \times \mathfrak{c}^{-1}\right)
$$

and this implies the invariance of $\mathfrak{z}_{\bullet}^{*}$. 
The point (ii) tells us that we have a well-defined bijection $\widetilde{\mathfrak{c}^{*}}: \mathfrak{g}^{*} / A d^{*} \rightarrow \mathfrak{g}^{*} / A d^{*}$. It can be shown that this map is compatible with the one acting on the level of the unitary dual:

$$
\widehat{\mathrm{c}}\left(\xi_{\Omega}\right)=\xi_{\mathfrak{c}^{*}(\Omega)}, \quad \forall \Omega \in \mathfrak{g}^{*} / \mathrm{Ad}^{*} .
$$

This combined with (iv), or a direct proof, shows in the admissible case that $\widehat{\mathrm{c}}\left(\widehat{G}_{\bullet}\right) \subset \widehat{G}_{\bullet}$.

Remark 7.2. Having the form $\Omega_{\mathcal{U}}=\mathcal{U}+\mathfrak{z}^{\dagger}$ for some $\mathcal{U} \in \mathfrak{z}_{\bullet}^{*}$, the flat orbits can be obtained from each other through translations. But these translations in $\mathfrak{g}^{*}$ are not corresponding to Lie algebra automorphisms of $\mathfrak{g}$ and they do not match our setting.

If we know in advance that two irreducible representations $\xi$ and $\eta$ are connected by an automorphism, i. e. $\eta=\widehat{c}(\xi)$, this is valuable: they both act on the same Hilbert space, and one can be easily constructed in terms of the other. Consequently, the Pedersen quantizations $\operatorname{Ped}_{\xi}$ and $\operatorname{Ped}_{\eta}$ are also directly connected. This is particularly effective when an automorphism group acts transitively on a relevant family of (classes of) irreducible representations. Under favorable circumstances, this can be used in the framework of the quantizations we studied on $\mathrm{G} \times \widehat{\mathrm{G}}$ and $\mathrm{G} \times \mathfrak{g}^{*}$ respectively.

Let us just explore the case when $\mathrm{G}$ is admissible and graded and the center $\mathfrak{z}$ of the Lie algebra is onedimensional. This happens for the Heisenberg groups and for the examples in Subsections 7.2 and 7.3, but it does not hold in Section 6. When this is the case, then $\mathfrak{z}^{*}$ is also one-dimensional and, since the Pfaffian is a homogeneous polynomal, it is easy to see that $\mathfrak{z}_{\bullet}^{*}=\mathfrak{z}^{*} \backslash\{0\}$. The dilation group on $\mathfrak{g}$ generates, as above, groups of dilations on $\mathfrak{g}^{*}, \mathfrak{g}^{*} / \mathrm{Ad}^{*}, \mathrm{G}, \widehat{\mathrm{G}}, \widehat{\mathrm{G}}_{\bullet}, \mathfrak{z}^{*}$ and $\mathfrak{z}_{\bullet}^{*}$. In particular, in $\mathfrak{z}_{\bullet}^{*} \cong \mathbb{R} \backslash\{0\}$ there are two orbits $\mathbb{R}_{ \pm}$. Another automorphism $\mathfrak{i n v}(X):=-X$ (or, equivalently, $\operatorname{inv}(x)=x^{-1}$ at the group level) connects the two orbits, because it acts as $\mathfrak{i n v}^{*}(\mathcal{Z})=-\mathcal{Z}$ on the dual of the center of $\mathfrak{g}$. Thus $1 \in \mathbb{R} \backslash\{0\}$ can be connected with any other element in $\mathfrak{z}_{\bullet}^{*}$ and one has

$$
\xi_{r}=\xi_{1} \circ \mathrm{dil}_{r} \text { if } r>0 \text { and } \xi_{r}=\xi_{1} \circ \mathrm{dil}_{r} \circ \text { inv if } r<0 .
$$

Consequently, in this case, if one of the generic irreducible representation (corresponding to one of the flat orbits) is computed, the others are easily generated using the dilations and eventually an inversion. We recall that, by abuse, representations has been identified with their unitary equivalence classes; thus, in terms of representations, (7.4) merely means equivalence and not equality.

\subsection{A five dimensional three-step graded admissible group}

For a first example [3, Ex. 5.7], the Lie algebra is generated by $\left\{E_{0}, E_{1}, E_{2}, E_{3}, E_{4}\right\}$, with the non-trivial brackets

$$
\left[E_{4}, E_{1}\right]=\left[E_{3}, E_{2}\right]=E_{0}, \quad\left[E_{4}, E_{3}\right]=E_{1} .
$$

So it can be seen as $\mathbb{R}^{5}$ with bracket

$$
\left[\left(q_{0}, q_{1}, q_{2}, q_{3}, q_{4}\right),\left(p_{0}, p_{1}, p_{2}, p_{3}, p_{4}\right)\right]:=\left(q_{4} p_{1}-q_{1} p_{4}+q_{3} p_{2}-q_{2} p_{3}, q_{4} p_{3}-q_{3} p_{4}, 0,0,0\right) .
$$

One has $[\mathfrak{g}, \mathfrak{g}]=\mathbb{R}^{2} \times\{(0,0,0)\} \equiv \operatorname{Span}\left\{E_{0}, E_{1}\right\}$ and the center is one-dimensional:

$$
\mathfrak{z}=[[\mathfrak{g}, \mathfrak{g}], \mathfrak{g}]=\mathbb{R} \times\{(0,0,0,0)\} \equiv \operatorname{Span}\left\{E_{0}\right\} .
$$

The Lie algebra $\mathfrak{g}$ is graded by

$$
\mathfrak{g}=\mathfrak{w}_{1} \oplus \mathfrak{w}_{2} \oplus \mathfrak{w}_{3}=\operatorname{Span}\left\{E_{3}, E_{4}\right\} \oplus \operatorname{Span}\left\{E_{1}, E_{2}\right\} \oplus \operatorname{Span}\left\{E_{0}\right\},
$$


so the dilations are

$$
\mathfrak{d i l}_{r}\left(q_{0}, q_{1}, q_{2}, q_{3}, q_{4}\right):=\left(r^{3} q_{0}, r^{2} q_{1}, r^{2} q_{2}, r q_{3}, r q_{4}\right), \quad r \in \mathbb{R}_{+} .
$$

Since this Lie algebra is not stratified, there is no sub-Laplacian to use. A Rockland operator can be computed by applying Remark 5.1. Our basis satisfies the assumptions, since $\left\{E_{2}, E_{3}, E_{4}\right\}$ generates $\mathrm{G}$ as a Lie algebra and $\left\{E_{0}, E_{1}\right\}$ generates $[\mathfrak{g}, \mathfrak{g}]$ linearly; thus $n=5$ and $n^{\prime}=3$. One may take $p=6$ and

$$
\mathcal{R}:=-E_{2}^{6}+E_{3}^{12}+E_{4}^{12}
$$

is a 12-homogeneous Rockland operator.

For the dual we set

$$
\mathfrak{g}^{*}:=\mathbb{R}^{5} \ni\left(\rho_{0}, \rho_{1}, \rho_{2}, \rho_{3}, \rho_{4}\right) \equiv \rho_{0} \mathcal{E}_{0}+\rho_{1} \mathcal{E}_{1}+\rho_{2} \mathcal{E}_{2}+\rho_{3} \mathcal{E}_{3}+\rho_{4} \mathcal{E}_{4} .
$$

The anihilator of the center is

$$
\mathfrak{z}^{\perp}=\{0\} \times \mathbb{R}^{4}=\operatorname{Span}\left\{\mathcal{E}_{1}, \mathcal{E}_{2}, \mathcal{E}_{3}, \mathcal{E}_{4}\right\},
$$

while the dual of the center identifies to

$$
\mathfrak{z}^{*} \equiv \mathbb{R} \times\{(0,0,0,0)\}=\operatorname{Span}\left\{\mathcal{E}_{0}\right\} .
$$

On the corresponding connected simply connected Lie group $G \equiv \mathfrak{g}=\mathbb{R}^{4}$ one has the multiplication

$$
\begin{aligned}
& \left(q_{0}, q_{1}, q_{2}, q_{3}, q_{4}\right) \bullet\left(p_{0}, p_{1}, p_{2}, p_{3}, p_{4}\right) \\
& =\left(q_{0}+p_{0}+\frac{1}{2}\left(q_{4} p_{1}-q_{1} p_{4}+q_{3} p_{2}-q_{2} p_{3}\right)+\frac{1}{12}\left(q_{4}-p_{4}\right)\left(q_{4} p_{3}-q_{3} p_{4}\right),\right. \\
& \left.\quad q_{1}+p_{1}+\frac{1}{2}\left(q_{4} p_{3}-q_{3} p_{4}\right), q_{2}+p_{2}, q_{3}+p_{3}, q_{4}+p_{4}\right) .
\end{aligned}
$$

One easily computes the coadjoint action

$$
\begin{aligned}
& \operatorname{Ad}_{\left.\left(q_{0}, q_{1}, q_{2}, q_{3}, q_{4}\right)\right)}^{*}\left(\rho_{0}, \rho_{1}, \rho_{2}, \rho_{3}, \rho_{4}\right) \\
& =\left(\rho_{0}, \rho_{1}-q_{4} \rho_{0}, \rho_{2}-q_{3} \rho_{0}, \rho_{3}+\left(q_{2}-q_{4}^{2} / 4\right) \rho_{0}-q_{4} \rho_{1}, \rho_{4}+\left(q_{1}+(1 / 4) q_{4} q_{3}\right) \rho_{0}+q_{3} \rho_{1}\right) .
\end{aligned}
$$

The fixed points (0-dimensional coadjoint orbits) are those situated in the subspace $\{(0,0)\} \times \mathbb{R}^{3}$. Other (2-dimensional) coadjoint orbits are

$$
\left(0, \rho_{1}, 0,0,0\right)+\{(0,0,0)\} \times \mathbb{R}^{2}, \quad \rho_{1} \in \mathbb{R} \backslash\{0\} .
$$

The flat (generic, 4-dimensional) coadjoint orbits have all the form

$$
\Omega_{\left(\rho_{0}, 0,0,0,0\right)} \equiv \Omega_{\rho_{0}}:=\left\{\rho_{0}\right\} \times \mathbb{R}^{4}=\left\{\left(\rho_{0}, 0,0,0,0\right)\right\}+\{0\} \times \mathbb{R}^{4}=\left\{\left(\rho_{0}, 0,0,0,0\right)\right\}+\mathfrak{z}^{\perp}
$$

for some fixed $\rho_{0} \neq 0$.

The canonical bilinear form reads

$$
\begin{aligned}
\operatorname{Bil}_{\left(\rho_{0}, \rho_{1}, \rho_{2}, \rho_{3}, \rho_{4}\right)} & \left(\left(q_{0}, q_{1}, q_{2}, q_{3}, q_{4}\right),\left(p_{0}, p_{1}, p_{2}, p_{3}, p_{4}\right)\right) \\
= & \left(q_{4} p_{1}-q_{1} p_{4}+q_{3} p_{2}-q_{2} p_{3}\right) \rho_{0}+\left(q_{4} p_{3}-q_{3} p_{4}\right) \rho_{1}
\end{aligned}
$$


and is non-degenerate when restricted to the common predual $\omega=\{0\} \times \mathbb{R}^{4} \subset \mathfrak{g}$ of the flat orbits if and only if $\rho_{0} \neq 0$. Now, if $\left(\rho_{0}, 0,0,0,0\right) \in \mathfrak{z}^{*}$, one has

$$
\operatorname{Bil}_{\left(\rho_{0}, 0,0,0,0\right)}\left(\left(q_{1}, q_{2}, q_{3}, q_{4}\right),\left(p_{1}, p_{2}, p_{3}, p_{4}\right)\right)=\left(q_{4} p_{1}-q_{1} p_{4}+q_{3} p_{2}-q_{2} p_{3}\right) \rho_{0},
$$

so

$$
\operatorname{Pf}^{2}\left(\rho_{0}\right) \equiv \operatorname{Pf}^{2}\left(\rho_{0}, 0,0,0,0\right)=\operatorname{det} \operatorname{Bil}_{\left(\rho_{0}, 0,0,0,0\right)}=\rho_{0}^{4},
$$

confirming once again that

$$
\mathfrak{z}_{\bullet}^{*}=\mathbb{R} \backslash\{0\} \equiv(\mathbb{R} \backslash\{0\}) \times\{(0,0,0,0)\} .
$$

Based on Proposition 2.3, the concrete Plancherel measure on $\mathfrak{z}_{\bullet}^{*}$ is

$$
d \mu\left(\rho_{0}\right)=8\left|\operatorname{Pf}\left(\rho_{0}\right)\right| d \rho_{0}=8\left|\rho_{0}\right|^{2} d \rho_{0} .
$$

\subsection{Another five dimensional three-step graded admissible group}

We present briefly a similar case, that is mentioned in [3, Ex. 5.8]; it is different, slightly more complicated, but still similar to the one treated above. The non-trivial structure equations are

$$
\left[E_{4}, E_{3}\right]=E_{2}, \quad\left[E_{4}, E_{2}\right]=E_{1}, \quad\left[E_{4}, E_{1}\right]=\left[E_{3}, E_{2}\right]=E_{0},
$$

corresponding to the bracket

$$
\left[\left(q_{0}, q_{1}, q_{2}, q_{3}, q_{4}\right),\left(p_{0}, p_{1}, p_{2}, p_{3}, p_{4}\right)\right]:=\left(q_{3} p_{2}-q_{2} p_{3}+q_{4} p_{1}-q_{1} p_{4}, q_{4} p_{2}-q_{2} p_{4}, q_{4} p_{3}-q_{3} p_{4}, 0,0\right) .
$$

The center $\mathfrak{z}=\operatorname{Span}\left(E_{0}\right)$ is once again one dimensional, so the relevant square integrable modulo the center irreducible representations are generated by dilations and the inversion from a given one; we leave their computation to the reader. The dilation is defined by the (non-stratified) grading

$$
\mathfrak{g}=\operatorname{Span}\left\{E_{4}\right\} \oplus \operatorname{Span}\left\{E_{3}\right\} \oplus \operatorname{Span}\left\{E_{2}\right\} \oplus \operatorname{Span}\left\{E_{1}\right\} \oplus \operatorname{Span}\left\{E_{0}\right\},
$$

i.e.

$$
\mathfrak{d i l}_{r}\left(E_{j}\right):=r^{5-j} E_{j}, \quad r>0, j=0,1,2,3,4 .
$$

Applying Remark 5.1 with $n=5, n^{\prime}=2, Z_{j}=E_{5-j}$ and $p=3 \cdot 4 \cdot 5=60$, one checks easily that

$$
\mathcal{R}:=E_{4}^{120}+E_{3}^{60}
$$

is a homogeneous Rockland operator of order 120 .

It is not difficult to see that the flat coadjoint orbits are labelled by $\rho_{0} \neq 0$ :

$$
\Omega_{\rho_{0}}=\left\{\rho_{0}, 0,0,0,0\right\}+\mathfrak{z}^{\perp}=\left\{\left(\rho_{0}, \rho_{1}, \rho_{2}, \rho_{3}, \rho_{4}\right) \mid \rho_{1}, \rho_{2}, \rho_{3}, \rho_{4} \in \mathbb{R}\right\} .
$$

The canonical bilinear form

$$
\begin{aligned}
\operatorname{Bil}_{\left(\rho_{0}, \rho_{1}, \rho_{2}, \rho_{3}, \rho_{4}\right)} & \left(\left(q_{0}, q_{1}, q_{2}, q_{3}, q_{4}\right),\left(p_{0}, p_{1}, p_{2}, p_{3}, p_{4}\right)\right) \\
= & \left(q_{3} p_{2}-q_{2} p_{3}+q_{4} p_{1}-q_{1} p_{4}\right) \rho_{0}+\left(q_{4} p_{2}-q_{2} p_{4}\right) \rho_{1}+\left(q_{4} p_{3}-q_{3} p_{4}\right) \rho_{2}
\end{aligned}
$$

is most relevant for $\mathcal{Z}=\left(\rho_{0}, 0,0,0,0\right) \in \mathfrak{z}^{*}$ and $X=\left(0, q_{1}, q_{2}, q_{3}, q_{4}\right), Y=\left(0, p_{1}, p_{2}, p_{3}, p_{4}\right) \in \omega$ (the commun predual of all the flat coadjoint orbits), leading to

$$
\operatorname{Bil}_{\rho_{0}}\left(\left(q_{0}, q_{1}, q_{2}, q_{3}, q_{4}\right),\left(p_{0}, p_{1}, p_{2}, p_{3}, p_{4}\right)\right)=\left(q_{3} p_{2}-q_{2} p_{3}+q_{4} p_{1}-q_{1} p_{4}\right) \rho_{0} .
$$

So, as in Subsection 7.2, the Plancherel measure on $\mathfrak{z}_{\bullet}^{*} \equiv \mathbb{R} \backslash\{0\}$ is

$$
d \mu\left(\rho_{0}\right)=8\left|\operatorname{Pf}\left(\rho_{0}\right)\right| d \rho_{0}=8 \operatorname{det} \operatorname{Bil}_{\left(\rho_{0}, 0,0,0,0\right)} d \rho_{0}=8\left|\rho_{0}\right|^{2} d \rho_{0} .
$$




\section{References}

[1] H. Bahouri, C. Fermanian-Kammerer and I. Gallagher: Phase Space Analysis and Pseudodifferential Calculus on the Heisenberg Group, Asterisque, 342, (2012).

[2] I. Beltiţă and D. Beltiţă: Continuity of Magnetic Weyl Calculs, J. Funct. Analysis, 260 (7), 1944-1968, (2011).

[3] I. Beltiţă and D. Beltiţă: Boundedness for Weyl-Pedersen Calculus on Flat Coadjoint Orbits, Int. Math. Res. Notices, 2015, 787-816, (2015).

[4] I. Beltiţă and D. Beltiţă: Smooth Vectors and Weyl-Pedersen Calculus for Representations of Nilpotent Lie Groups, Annals of the University of Bucharest (mathematical series) 1 (LIX), no. 1, 17-46 (2010).

[5] D. Burde: Characteristically Nilpotent Lie Algebras and Symplectic Structures, Forum Math. 18 (5), 769-787, (2006).

[6] L. J. Corwin and F. P. Greenleaf: Representations of Nilpotent Lie Groups and Applications, Cambridge Univ. Press, 1990.

[7] G. van Dijk, K.-H. Neeb, H. Salmasian and C. Zellner: On the Characterization of Trace Class Representations and Schwartz Operators, arXiv:1512.02451.

[8] K. Dykema, J. Noles, F. Sukochev and D. Zanin: On Reduction Theory and Brown Measure for Closed Unbounded Operators, arXiv: 1509.03362v1.

[9] J. Dixmier: Les $C^{*}$-algèbres et leurs représentations, Cahiers scientifiques, XXIX, Gauthier-Villars Éditeurs, Paris, 1969.

[10] V. Fischer and M. Ruzhansky: A Pseudo-differential Calculus on Graded Nilpotent Groups, in Fourier Analysis, pp. 107-132, Trends in Mathematics, Birkhäuser, 2014. arXiv: 1209.2621v2.

[11] V. Fischer and M. Ruzhansky: A Pseudo-differential Calculus on the Heisenberg Group, C. R. Acad. Sci. Paris, Ser I, 352, 197-204, (2014).

[12] V. Fischer and M. Ruzhansky: Quantization on Nilpotent Lie Groups, Progress in Mathematics, Vol. 314, Birkhäuser, 2016.

[13] G. B. Folland: Harmonic Analysis in Phase Space, Annals of Mathematics Studies, 122. Princeton University Press, Princeton, NJ, 1989.

[14] G. B. Folland and E. M. Stein: Hardy Spaces on Homogeneous Groups, Mathematical Notes, 28, Princeton Univ. Press, Princeton N. J. 1982.

[15] H. Führ: Abstract Harmonic Analysis of Continuous Wavelet Transforms, L.N.M. 1863, Sprincer-Verlag Berlin Heidelberg, 2005.

[16] D. Geller: Fourier analysis on the Heisenberg group. I. Schwartz space. J. Funct. Anal. 36, 205-254, (1980).

[17] P. Głowacki: A Symbolic Calculus and $L^{2}$-Boundedness on Nilpotent Lie Groups, J. Funct. Anal. 206, 233-251, (2004). 
[18] P. Głowacki: The Melin Calculus for General Homogeneous Groups, Ark. Mat., 45, no. 1, 31-48, (2007).

[19] P. Głowacki: Invertibility of Convolution Operators on Homogeneous Groups, Rev. Mat. Iberoam. 28, no. 1, 141-156, (2012).

[20] B. Helffer and J. Nourrigat: Caracterisation des opérateurs hypoelliptiqes homogènes invariants à gauche sur un groupe de Lie nilpotent gradué, Comm. P. D. E. 4 (8), 899-958, (1979).

[21] R. Howe: On the Connection Between Nilpotent Groups and Oscillatory Integrals Associated to Singularities, Pac. J. Math. 73, 329-363, (1977).

[22] A. A. Kirillov: Lectures on the Orbit Method, Graduate Studies in Mathematics, 64, American Mathematical Society, Providence, RI, 2004.

[23] J. Ludwig: Good Ideals in the Group Algebra of a Nilpotent Group, Math. Zeitsch. 16, 195-210 (1978).

[24] D. Manchon: Formule de Weyl pour les groupes de Lie nilpotentes, J. Reine Angew. Mat. 418, 77-129, (1991).

[25] D. Manchon: Calcul symbolyque sur les groupes de Lie nilpotentes et applications, J. Funct. Anal. 102 (2), 206-251, (1991).

[26] M. Măntoiu and M. Ruzhansky: Pseudo-differential Operators, Wigner Transform and Weyl Systems on Type I Locally Compact Groups, Preprint ArXiv and submitted.

[27] A. Melin: Parametrix Constructions for Right Invariant Differential Operators on Nilpotent Groups, Ann. Global Anal. Geom. 1 (1), 79-130, (1983).

[28] K. Miller: Invariant Pseudodifferential Operators on Two Step Nilpotent Lie Groups, Michigan Math. J. 29, 315-328, (1982).

[29] K. Miller: Inverses and Parametrices for Right-Invariant Pseudodifferential Operators on Two-Step Nilpotent Lie Groups, Trans. of the AMS, 280 (2), 721-736 (1983).

[30] C. C. Moore and J. Wolf: Square Integrable Representations of Nilpotent Groups, Trans. AMS, 185, 445-462, (1973).

[31] A. E. Nussbaum: Reduction Theory for Unbounded Closed Operators in Hilbert Space, Duke Math. J. 31, 33-44, (1964).

[32] N.V. Pedersen: Matrix Coefficients and a Weyl Correspondence for Nilpotent Lie Groups. Invent. Math. 118, 1-36, (1994).

[33] F. Ricci: Sub-Laplacians on Nilpotent Lie Groups, Unpublished Lecture Notes, accessible at http://homepage.sns.it/fricci/corsi.html.

[34] M. Ruzhansky and V. Turunen: Pseudodifferential Operators and Symmetries, Pseudo-Differential Operators: Theory and Applications 2, Birkhäuser Verlag, 2010.

[35] M. Ruzhansky and V. Turunen: Global quantization of pseudo-differential operators on compact Lie groups, SU(2) and 3-sphere. Int. Math. Res. Not. IMRN 11, 2439-2496, (2013). 